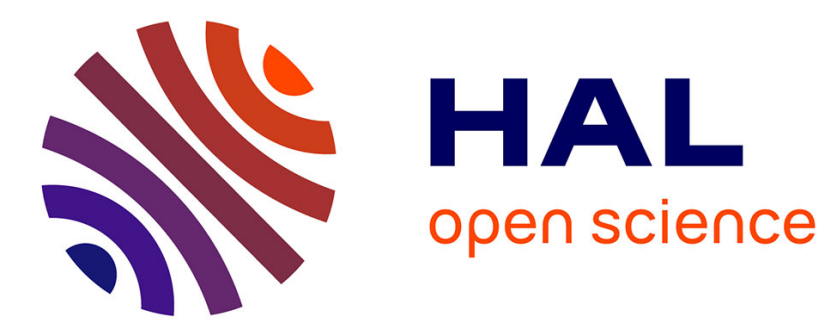

\title{
Des hommes médicalement assistés pour procréer: IAD, FIV, ICSI, bilan d'une révolution dans la prise en charge médicale de l'infertilité masculine
}

Elise de La Rochebrochard

\section{- To cite this version:}

Elise de La Rochebrochard. Des hommes médicalement assistés pour procréer: IAD, FIV, ICSI, bilan d'une révolution dans la prise en charge médicale de l'infertilité masculine. Population (édition française), 2003, 58 (4-5), pp.549-586. 10.3917/popu.304.0549 . hal-02270347

\section{HAL Id: hal-02270347 \\ https://hal.science/hal-02270347}

Submitted on 24 Aug 2019

HAL is a multi-disciplinary open access archive for the deposit and dissemination of scientific research documents, whether they are published or not. The documents may come from teaching and research institutions in France or abroad, or from public or private research centers.
L'archive ouverte pluridisciplinaire HAL, est destinée au dépôt et à la diffusion de documents scientifiques de niveau recherche, publiés ou non, émanant des établissements d'enseignement et de recherche français ou étrangers, des laboratoires publics ou privés. 


\title{
Des hommes médicalement assistés pour procréer
}

\author{
IAD, FIV, ICSI, bilan d'une révolution \\ dans la prise en charge médicale \\ de l'infertilité masculine
}

\author{
Élise de LA ROCHEBROCHARD*
}

\begin{abstract}
Au début des années 1980, l'apparition de la fécondation in vitro (FIV) a permis de traiter l'infertilité féminine. Les problèmes masculins avaient alors pour réponse essentielle l'insémination artificielle avec donneur (IAD) qui, en France, a néanmoins toujours suscité d'importantes réserves parmi les hommes, comme parmi les femmes. Élise de LA ROCHEBROCHARD montre qu'un tournant a été pris dans les années 1990 avec l'émergence et l'expansion très rapide d'une nouvelle technique, l'ICSI (injection intra-cytoplasmique de spermatozoïde), adaptée au traitement de l'infertilité masculine. L'ICSI a fait reculer le recours à l'IAD, et a stabilisé l'utilisation de la FIV " traditionnelle». Cependant, avec l'ICSI, la prise en charge de l'infertilité masculine continue à se faire dans le corps des femmes. Le développement rapide de cette nouvelle technique, dont toutes les conséquences n'ont pas encore été pleinement évaluées, soulève d'importants problèmes qui sont abordés dans cet article.
\end{abstract}

Avec la large diffusion des méthodes contraceptives médicalisées (Leridon et al., 1987; Leridon et al., 2002), les femmes peuvent avoir l'impression d'avoir concrétisé le célèbre slogan féministe des années 1970 : «Un enfant si je veux, quand je veux! ». Pourtant, une fois prise la décision d'avoir un enfant, une fois la contraception arrêtée, le couple perd la maîtrise des événements et l'enfant peut ne pas arriver. Pour 100 jeunes couples qui tentent l'aventure, 20 à 25 débutent dès le premier mois une grossesse qui mènera à la naissance d'un enfant vivant, 65 à 70 conçoivent durant les six premiers mois et 80 à 85 durant la

* Institut national d'études démographiques et Institut national de la santé et de la recherche médicale, Paris. 
première année (Spira, 1986; de Mouzon et al., 1988; Leridon, 1991). Au bout de cette année d'attente, il apparaît légitime pour les 15 à 20 couples n'ayant pas encore conçu d'aller consulter un médecin afin de discuter de cette infécondité involontaire.

Dans les faits, les couples peuvent s'inquiéter bien avant cette période d'une année. À partir de l'enquête Situations familiales et emploi, réalisée par l'Ined et l'Insee, on peut mesurer cette inquiétude à partir de deux indicateurs ${ }^{(1)}$. D'une part, les femmes étaient interrogées sur l'existence de difficultés perçues à partir de la question : "Vous est-il arrivé de mettre plus de temps que vous ne l'auriez voulu pour être enceinte et d'y parvenir finalement? ». Parmi les femmes ayant fait part de telles difficultés pour obtenir une grossesse, $30 \%$ ont déclaré avoir conçu avant la fin de la première année de tentative (tandis que $19 \%$ ont conçu au bout d'une année et $51 \%$ après un délai supérieur à un an). Le deuxième indicateur porte sur le délai de consultation, question posée aux femmes mais également aux hommes. Parmi les personnes ayant consulté un médecin pour infécondité (elles ou/et leur partenaire), $41 \%$ ont consulté alors qu'elles et leur partenaire cherchaient à concevoir depuis moins d'une année (tandis que $31 \%$ déclarent avoir consulté à la date du premier anniversaire et $28 \%$ après ce premier anniversaire) ${ }^{(2)}$. Ces deux indicateurs confirment l'hypothèse d'une « impatience » croissante des couples, hypothèse avancée à partir de l'analyse comparative des enquêtes de fécondité de 1978 et de 1988, qui a été publiée sous le titre «Stérilité, hypofertilité : du silence à l'impatience ? » (Leridon, 1991).

Il y a quelques décennies, l'infécondité de ces couples aurait probablement été considérée comme une « histoire de femmes »(3). Aujourd'hui, les couples doivent également envisager que leurs difficultés puissent avoir une origine masculine. Cette question de l'origine de l'infertilité a été explorée lors d'une enquête auprès de couples consultant un médecin pour infécondité entre juillet 1988 et juin 1989 en Indre-et-Loire, LoireAtlantique et Haute-Vienne (Thonneau et al., 1991). Les investigations médicales menées sur ces couples ont mis en évidence une anomalie du sperme dans trois cas sur cinq (tableau 1). Il s'agissait principalement d'une anomalie des caractéristiques dites conventionnelles du sperme (numération, mobilité, morphologie). Néanmoins, d'autres critères d'évaluation de la qualité du sperme sont discutés et certains suggèrent qu'une partie des infécondités idiopathiques (c'est-à-dire inexpliquées) pourraient bien être d'origine masculine, l'homme présentant une altération du sperme non mesurée par les critères conventionnels (Irvine, 1998)(4).

(1) Données non publiées. Cette enquête a été réalisée en 1994 sur un échantillon représentatif d'hommes $(n=1966)$ et de femmes $(n=3007)$ âgés de 18 à 49 ans.

(2) Les femmes déclarent plus souvent que les hommes avoir consulté avant la fin de la première année (44\% des femmes contre $37 \%$ des hommes, $p=0,3 \%$ ).

(3) L'anthropologue Françoise Héritier-Augé indique que dans presque toutes les sociétés humaines, la femme est considérée comme responsable de la stérilité (Héritier-Augé, 1985, p. 12). 
Quoi qu'il en soit, en matière d'infertilité, la recherche médicale d'un « coupable » semble insuffisante : dans deux cas sur cinq, l'investigation médicale met en évidence une cause à la fois féminine et masculine (tableau 1). Ce résultat a conduit les auteurs de l'enquête française à conclure que l'infertilité est avant tout une « histoire de couple ».

La part des infécondités masculines pourrait augmenter dans les années à venir. En effet, des recherches mettent en évidence une altération des caractéristiques spermatiques conventionnelles depuis une cinquantaine d'années dans les pays développés (Carlsen et al., 1992; de Mouzon et al., 1996). Ces altérations, à la fois quantitatives et qualitatives, conduisent à s'interroger sur une possible augmentation des infécondités masculines dans les jeunes générations.

Après avoir décrit le système de collecte des données sur les fécondations assistées en France et dans le reste du monde, cet article analyse la prise en charge médicale de l'infertilité masculine à travers les différentes techniques d'assistance médicale à la procréation. D'un côté, la fécondation in vitro obtient des taux de succès très faibles dans le cas d'une infertilité masculine sévère (cette technique ayant été initialement développée pour répondre aux infertilités féminines). De l'autre, l'insémination artificielle avec donneur a des taux de succès satisfaisants mais le couple doit alors accepter l'utilisation du sperme d'un donneur. Entre ces deux techniques, la prise en charge médicale de l'infertilité masculine apparaissait donc problématique jusqu'à l'apparition de l'injection intracytoplasmique de spermatozoïde, il y a une dizaine d'années. Nous verrons que si cette nouvelle technique a connu un essor important, sa large diffusion ne va pas sans soulever nombre de questions.

(4) D. Stewart Irvine (1998) discute les difficultés à diagnostiquer les infertilités masculines. Généralement, l'infertilité masculine est diagnostiquée à partir des caractéristiques spermatiques dites conventionnelles (numération, mobilité, morphologie) qui sont comparées à des critères de normalité reconnue (Crosignani et al., 1994; van den Eede, 1995). Cependant, cette procédure présente différentes limites : (i) il existe une variabilité des caractéristiques spermatiques d'un prélèvement à l'autre pour un même homme; (ii) l'évaluation des caractéristiques spermatiques est marquée par une certaine subjectivité qui s'exprime en particulier par une variabilité des résultats selon les laboratoires d'analyse; (iii) il existe des critères de normalité du sperme publiés par 1'OMS mais leur pertinence en termes de diagnostic de l'infertilité masculine est discutée. 
TABLEAU 1.- ORIGINE DE L'INFERTILITÉ DES COUPLES

$\left.\begin{array}{|l|r|}\hline \multicolumn{1}{|c|}{\text { Origine de l'infertilité }} & \text { Distribution } \\ \hline \text { Masculine uniquement } & 20 \% \\ \text { Masculine et féminine } & 38 \%\end{array}\right\} 58 \%$ d'infertilité masculine dont $\left\{\begin{array}{c}9 \% \text { azoospermique } \\ 49 \% \text { oligo - térato - asthénospermie } \\ \text { Féminine uniquement } \\ \text { Idiopathique (inexpliquée) }\end{array}\right.$

Tतs

के

5

$\pi$

욤

预 


\section{Systèmes de collecte de données sur les fécondations assistées}

En réponse aux problèmes d'infécondité se sont développées des techniques de fécondation in vitro (FIV). La FIV consiste à prélever des ovocytes (lors d'une ponction folliculaire) et à recueillir des spermatozoïdes - chez les conjoints en général, chez des donneurs parfois - pour réaliser la fécondation en laboratoire. Une fois l'ovocyte fécondé et devenu embryon, ce dernier est placé dans l'utérus ou dans les trompes de Fallope de la femme. C'est ainsi qu'est née Louise Brown le 25 juillet 1978 dans les faubourgs de Manchester (Angleterre), connue sous le nom de «premier bébé-éprouvette » (Steptoe et Edwards, 1978). La France attendra le 24 février 1982 pour voir naître à l'hôpital Antoine-Béclère de Clamart son «premier bébé-éprouvette », prénommé Amandine (Testart et Frydman, 1982).

\section{Diffusion de la FIV en France...}

Dans les années qui suivent la naissance d'Amandine, on observe une progression rapide du nombre de FIV réalisées en France et l'on atteint un niveau de l'ordre de 5000 bébés-éprouvette par an dans les années 1990-1992.

Face à cette nouvelle technique médicale, des interrogations surgissent. Il apparaît en particulier nécessaire d'évaluer les risques potentiels pour l'enfant et pour la femme (Breart et de Mouzon, 1995). C'est donc à la fois dans un souci de santé publique et de recherche que, dès 1986, la France met en place une enquête prospective intitulée FIVNAT, pour FIV-NATional ${ }^{(5)}$ (de Mouzon et al., 1993; FIVNAT, 1993). Cette enquête repose sur une collecte d'informations exhaustive dans des centres de FIV volontaires pour y participer. Deux fiches sont recueillies. La fiche « tentative » décrit le bilan d'infécondité du couple et le déroulement de la tentative de FIV (ponction folliculaire, transfert d'embryon(s), obtention ou non d'une grossesse). La fiche « grossesse » décrit le déroulement médical de la grossesse, son issue et l'état de santé de(s) (l')enfant(s) à la naissance. Entre 1986 et 2000, FIVNAT a enregistré plus de 340000 fiches « tentatives » et plus de 29000 fiches « grossesses ».

Parallèlement à FIVNAT, un système de collecte national a été mis en place afin d'obtenir une estimation exhaustive du nombre de FIV réali-

(5) FIVNAT est une association dirigée par un bureau élu tous les deux ans lors du congrès de la Fédération française d'études en reproduction (FFER). Le codage, le contrôle, le traitement et l'analyse des données sont réalisés à l'hôpital du Kremlin-Bicêtre (94) au sein de l'unité mixte Inserm-Ined, sous la direction d'un épidémiologiste, le docteur Jacques de Mouzon (Inserm). Chaque année, un bilan est publié ainsi que des analyses plus spécifiques. Pour une présentation de l'association FIVNAT et des résultats générés par cette enquête, le lecteur peut consulter le site Internet de FIVNAT à l'adresse suivante : http://perso.wanadoo.fr/fivnat.fr/. 
sées en France. Ce système se fonde sur les bilans annuels d'activité des centres, qui ont été initialement collectés dans le cadre du GEFF (Groupement de l'étude des fécondations in vitro en France)(6) et le sont désormais dans le cadre d'une déclaration obligatoire au ministère de la Santé (7). Les bilans d'activité doivent en outre rendre compte du devenir des grossesses obtenues par FIV (et donc du nombre d'enfants qui en sont issus), mais ce système se heurte à la difficulté du retour des informations entre les maternités où naissent les enfants et les centres de FIV. On estime en particulier que le nombre de naissances déclaré dans les bilans d'activité est sous-estimé.

L'utilisation croisée de ces deux sources (enquête FIVNAT et bilans annuels d'activité des centres de FIV) permet d'estimer l'activité FIV française : les bilans d'activité fournissent une mesure exhaustive du nombre de cycles de fécondation assistée réalisés en France et l'enquête FIVNAT est utilisée pour mesurer les taux de succès (grossesses, accouchements, enfants) en supposant que les taux de succès des centres participant à FIVNAT sont identiques à ceux de l'ensemble des centres. Les conséquences de cette approximation pourraient être une légère surestimation du nombre d'enfants nés par FIV en France; mais on peut juger cette approximation satisfaisante au vu du fort taux de couverture de l'enquête FIVNAT qui a enregistré $92 \%$ des ponctions réalisées en France sur la période 1995-1999 (84\% sur la période 1986-1999)(8).

De 1982 à 1999, plus de 66500 accouchements ont été obtenus grâce à une FIV, donnant lieu à la naissance de plus de 85000 enfants (tableau 2)(9). Ces chiffres reflètent le taux important de naissances multiples : 127 à 130 enfants naissent pour 100 accouchements (FIVNAT, 1995). Le développement de la FIV est une des causes de la progression des naissances multiples en France : alors qu'un accouchement sur 100 donnait naissance à des jumeaux entre 1901 et 1970, c'est le cas d'un accouchement sur 75 dans les années 1990 (Daguet, 2002). Les autres éléments explicatifs de la croissance des naissances multiples sont le développement des stimulations hormonales, la meilleure prise en charge médicale des grossesses multiples et, dans une moindre mesure, l'augmentation des naissances chez les femmes de 35-39 ans. Ces taux élevés de

(6) Les bilans du GEFF ont été publiés chaque année dans le ${ }^{\circ}$ 7-8 de Contraception, Fertilité, Sexualité entre 1987 et 1992.

(7) Loi $\mathrm{n}^{\circ}$ 94-654 du 29 juillet 1994 relative au don et à l'utilisation des éléments et produits du corps humain, à l'assistance médicale à la procréation et au diagnostic prénatal. Article L. 184-2 : «Tout établissement ou laboratoire autorisé à pratiquer des activités d'assistance médicale à la procréation ou de diagnostic prénatal, tout centre pluridisciplinaire de diagnostic prénatal est tenu de présenter au ministre chargé de la santé un rapport annuel d'activité suivant des modalités déterminées par arrêté de ce ministre. Il est également tenu d'établir et de conserver dans des conditions fixées par décret en Conseil d'État des registres relatifs aux gamètes et aux embryons qu'il conserve ».

(8) Le taux de couverture de FIVNAT était de $61 \%$ lors de la première année de collecte en 1986 et a atteint $94 \%$ à partir de 1993.

(9) Les ponctions déclarées dans le tableau 2 concernent les ponctions réalisées dans le cadre des FIV conventionnelles mais également des ICSI (voir plus loin). 
TABLEAU 2.- BILAN DES FÉCONDATIONS MÉDICALEMENT ASSISTÉES EN FRANCE DE 1982 À 1999 (ESTIMATIONS)

\begin{tabular}{|c|c|c|c|c|c|c|}
\hline \multirow{2}{*}{ Année } & \multirow{2}{*}{ Centres agréés } & \multicolumn{2}{|c|}{ Cycles(a) $^{(a)}$} & \multirow{2}{*}{$\begin{array}{l}\text { Grossesses } \\
\text { cliniques }^{(d)}\end{array}$} & \multirow{2}{*}{ Accouchements $^{(\mathrm{d})}$} & \multirow{2}{*}{ Enfants $^{(\mathrm{d})}$} \\
\hline & & Ponctions $(b)$ & $\mathrm{TEC}^{(\mathrm{c})}$ & & & \\
\hline $1982-1985^{(\mathrm{e})}$ & - & 12000 & - & 1400 & 900 & 1000 \\
\hline 1986 & 55 & 11779 & - & 1767 & 1413 & 1838 \\
\hline 1987 & 92 & 18617 & 1409 & 3252 & 2370 & 3065 \\
\hline 1988 & 121 & 23143 & 1840 & 4251 & 3124 & 4040 \\
\hline 1989 & 115 & 25648 & 2137 & 4841 & 3489 & 4511 \\
\hline 1990 & 130 & 27963 & 2682 & 5578 & 4055 & 5239 \\
\hline 1991 & 109 & 27498 & 2827 & 5174 & 4007 & 5178 \\
\hline 1992 & 102 & 26063 & 2728 & 5172 & 3792 & 4893 \\
\hline 1993 & 95 & 26140 & 2784 & 5660 & 4082 & 5269 \\
\hline 1994 & 97 & 28920 & 3478 & 6069 & 4639 & 5989 \\
\hline 1995 & 101 & 33000 & 4194 & 7007 & 5173 & 6661 \\
\hline 1996 & 88 & 35325 & 5173 & 8425 & 6158 & 7890 \\
\hline 1997 & 94 & 38752 & 6686 & 9345 & 6925 & 8797 \\
\hline 1998 & 96 & 39800 & 7000 & 9617 & 8187 & 10331 \\
\hline 1999 & 96 & 41000 & 7500 & 10808 & 8462 & 10675 \\
\hline $\operatorname{Total}^{(\mathrm{f})}$ & & 415648 & 50438 & 88366 & 66776 & 85376 \\
\hline \multicolumn{7}{|c|}{$\begin{array}{l}\text { (a) Les nombres de cycles (ponctions et TEC) sont issus des bilans d'activité des centres. Ces bilans d'activité étaient dans un premier temps relevés dans le cadre de } \\
\text { l'enquête GEFF (Groupement de l'étude des fécondations in vitro en France) et le sont désormais dans le cadre d'une déclaration obligatoire au ministère de la Santé. Les } \\
\text { premiers bilans ont été publiés dans la revue Contraception, Fertilité, Sexualité. Les données non publiées ont été obtenues auprès du docteur Jacques de Mouzon } \\
\text { (Inserm), chargé d'établir les bilans annuels. } \\
\text { (b) Ponctions réalisées dans le cadre d'une technique de fécondation médicalement assistée quelle qu'elle soit, les deux principales étant la FIV classique et l'ICSI (injec- } \\
\text { tion intra-cytoplasmique de spermatozoïde). } \\
\text { (c) TEC = transfert d'embryons congelés. Lors d'une tentative de FIV, une partie des embryons peuvent être congelés afin d'être transférés lors d'une tentative ultérieure. } \\
\text { La congélation d'embryons est en particulier proposée lorsque le nombre d'embryons obtenus est supérieur au nombre d'embryons transférés. } \\
\text { (d) Les nombres de grossesses, d'accouchements et d'enfants indiqués dans les bilans d'activité des centres sont sous-estimés car une partie des grossesses sont perdues } \\
\text { de vue avant leur terme. À partir de } 1986 \text {, les chiffres présentés ici sont une estimation basée sur l'hypothèse d'un taux de succès en France identique à celui observé dans } \\
\text { l'enquête FIVNAT. Par exemple, en } 1990 \text {, on observe dans FIVNAT un taux de grossesses par ponction de } 18,7 \% \text { et un taux de grossesses par TEC de } 13,0 \% \text {, d'où } \\
\text { l'estimation du nombre de grossesses en France }: 27963 \text { ponctions } \times 0,187+2682 \text { TEC } \times 0,130=5578 \text { grossesses. } \\
\text { (e) Pour la période } 1982-1985 \text {, il s'agit d'une estimation agrégée pour les } 4 \text { années. } \\
\text { (f) Pour la période } 1982-1999, \text { les } 415648 \text { ponctions ont abouti à } 81445 \text { grossesses, } 62022 \text { accouchements, } 79815 \text { enfants. Les } 50438 \text { TEC ont abouti à } 6921 \text { gros- } \\
\text { sesses, } 4754 \text { accouchements, } 5561 \text { enfants. } \\
\text { Sources : voir notes ci-dessus. }\end{array}$} \\
\hline
\end{tabular}


grossesses multiples ont des conséquences importantes pour les enfants : elles se traduisent par un risque élevé de prématurité et donc de morbidité (Epelboin et Blondeau, 1989; Laborie, 1994a ; Dehan, 1998; Olivennes, 2001 ; Papiernik et de Mouzon, 2002).

\section{2. ... et dans le reste du monde}

Qu'en est-il dans le reste du monde? Depuis vingt-cinq ans, cinq bilans mondiaux de l'activité FIV ont été publiés par le Groupe de travail international pour les registres sur la reproduction assistée (IWGROAR : International Working Group on Assisted Reproduction) puis par le Comité international pour la surveillance des techniques d'assistance à la reproduction (ICMART : International Committee on Monitoring of Assisted Reproductive Techniques). Ils rapportent l'activité de quelques dizaines de pays pour les années 1989, 1991, 1993, 1995 et 1998(10). Le dernier bilan mondial repose en partie sur les informations fournies par les trois registres régionaux existants : le registre européen, le registre pour l'Australie et la Nouvelle-Zélande et le registre d'Amérique latine. En tout, une quarantaine de pays ont participé au bilan 1998, dont $59 \%$ ont déclaré exhaustivement leur activité.

Les FIV sont essentiellement effectuées dans les pays développés. Cependant, l'Organisation mondiale de la santé a récemment reçu une demande de conseils pour la mise en place et l'utilisation des techniques d'assistance à la reproduction, les «ART» (Assisted Reproductive Techniques), de la part des pays en voie de développement où l'offre d'ART émane essentiellement de cliniques privées (Vayena et al., 2002). Certains pays développés, comme le Japon, ont connu une diffusion très rapide de la FIV ces dernières années : entre 1995 et 1998, le nombre de centres de FIV a augmenté de manière exponentielle dans ce pays, passant de 96 à 425. Grâce à cette importante expansion, le Japon a réalisé au cours de l'année 1998 plus de 53000 ponctions, ce qui le place entre les États-Unis, qui réalisent le plus grand nombre de ponctions (plus de 60000 ), et les trois grands pays européens (France, Allemagne, RoyaumeUni), qui ont réalisé chacun environ 40000 ponctions durant l'année 1998. L'Europe est, de loin, le continent qui a le plus recours à la fécondation assistée.

\footnotetext{
(10) Ces rapports ont été publiés dans les actes des colloques au cours desquels ils ont été présentés : les congrès mondiaux sur «la fécondation in vitro et les techniques d'assistance » pour les bilans de 1989 (Paris, France, juin 1991), de 1991 (Kyoto, Japon, septembre 1993) et de 1997 (Vancouver, Canada, mai 1997); les congrès mondiaux sur « la fertilité et la stérilité » pour les bilans de 1993 (Montpellier, France, septembre 1995) et de 1998 (Melbourne, Australie, novembre 2001).
} 


\section{Recours à la FIV en Europe}

En Europe, la Société européenne de reproduction humaine et d'embryologie (ESHRE) a proposé en 1999 un programme pour regrouper les données sur les FIV à partir des informations collectées dans chaque pays. Ce programme a déjà donné lieu à la publication de deux bilans pour les années 1997 et 1998 (Nygren et Andersen, 2001a et 2001b). En 1998, 18 pays ont participé au programme (tableau 3). Cependant, seuls 9 pays ont fourni des données exhaustives : si l'Europe septentrionale dispose de registres nationaux exhaustifs, ce n'est pas le cas de l'Europe méridionale et orientale, tandis que l'Europe occidentale est divisée entre ces deux groupes. Par ailleurs, les auteurs du bilan notent une variabilité de la qualité des données déclarées (taux de couverture très variables, taux de grossesses perdues de vue différents avec des pays comme la Suisse où l'information sur les accouchements et les enfants reste très parcellaire, définitions hétérogènes selon les pays, etc.). Face à ces difficultés, le consortium européen travaille actuellement à l'amélioration du système de collecte; pour le moment, une analyse comparative de la diffusion de la FIV en Europe reste donc difficile.

On peut cependant estimer une fourchette du nombre de cycles réalisés dans les pays n'ayant pas de données exhaustives (tableau 3). Pour ces pays, le nombre de cycles enregistrés constitue la borne inférieure du nombre de cycles réalisés; par ailleurs, nous avons fait l'hypothèse que l'activité des centres non enregistrés dans le bilan était identique à l'activité des centres enregistrés. On surestime probablement ainsi l'activité FIV (en particulier pour les pays ayant un faible taux de couverture comme l'Espagne) et nous avons considéré que cette estimation constituait la borne supérieure du nombre de cycles réalisés dans le pays. Si on rapporte le nombre de cycles ainsi estimé au nombre de femmes âgées de 15 à 49 ans dans chaque pays, on dispose d'un indicateur du taux de recours à la FIV. Ce taux varie entre 1 (en Russie) et 66 (au Danemark) pour 10000 femmes d'âge fécond. En France, le taux de recours à la FIV est de 32 cycles pour 10000 femmes d'âge fécond, proche de la valeur observée aux Pays-Bas, en Finlande et en Norvège. En Allemagne, en Suisse et au Royaume-Uni, les taux de recours à la FIV sont plus faibles (de l'ordre de 23 à 28 pour 10000 ), tandis qu'en Belgique, au Danemark, en Suède et en Islande, ils sont plus élevés (supérieurs à 40 pour 10000 ).

En adoptant une démarche similaire, on peut estimer une fourchette du pourcentage d'enfants nés par fécondation assistée parmi les enfants nés dans chaque pays (tableau 3). Au cours de l'année 1999, 1,4\% des enfants sont nés par fécondation assistée en France. Le pourcentage d'enfants nés par FIV est fortement lié au nombre de cycles réalisés pour 10000 femmes (figure 1a) : ce pourcentage augmente de $1 \%$ lorsque le nombre de cycles réalisés pour 10000 femmes d'âge fécond augmente de 20 unités. 
TABLEAU 3.- FÉCONDATIONS MÉDICALEMENT ASSISTÉES DANS 18 PAYS EUROPÉENS POUR L'ANNÉE 1998 (ESTIMATIONS)

\begin{tabular}{|c|c|c|c|c|c|c|c|}
\hline Pays & $\begin{array}{c}\text { \% de centres } \\
\text { enregistrés }\end{array}$ & $\begin{array}{c}\text { Nombre } \\
\text { de cycles } \\
\text { (a) }\end{array}$ & $\mid \begin{array}{l}\text { Nombre de cycles } \\
\text { p. } 10000 \text { femmes } \\
\text { de } 15-49 \text { ans }\end{array}$ & $\begin{array}{l}\text { \% d'enfants nés } \\
\text { par fécondation } \\
\text { assistée }(\mathrm{b})\end{array}$ & $\begin{array}{l}\text { Indice synthé- } \\
\text { tique de fécondité } \\
\text { (enfants } \\
\text { par femme) }\end{array}$ & $\begin{array}{l}\text { Taux d'infécon- } \\
\text { dité définitive } \\
\text { dans la généra- } \\
\text { tion } 1955\end{array}$ & $\begin{array}{l}\text { Dépenses de } \\
\text { santé par habi- } \\
\text { tant en } 1998 \\
(\text { SPA })^{(c)}\end{array}$ \\
\hline \multicolumn{8}{|l|}{ Europe méridionale } \\
\hline Espagne & $38 \%$ & $8771-23124$ & $9-23$ & $0,5-1,3$ & 1,16 & 9,2 & 1194 \\
\hline Grèce & $44 \%$ & $6973-15689$ & $27-60$ & $1,6-3,7$ & 1,29 & 8,6 & 1198 \\
\hline Italie & $\sim 54 \%$ & $13341-24706$ & $9-18$ & $0,5-1,0$ & 1,17 & 12,7 & 1824 \\
\hline Portugal & Inconnu & $1217-?$ & $5-?$ & $0,1-?$ & 1,46 & 9,7 & 1203 \\
\hline \multicolumn{8}{|l|}{ Europe orientale } \\
\hline Hongrie & $75 \%$ & $2094-2792$ & $8-11$ & $0,5-0,7$ & 1,33 & 8,5 & - \\
\hline République tchèque & $93 \%$ & $7879-8442$ & $30-32$ & $1,9-2,1$ & 1,16 & 6,2 & - \\
\hline Russie & $63 \%$ & $4514-7222$ & $1-2$ & $0,1-0,1$ & 1,24 & - & - \\
\hline \multicolumn{8}{|l|}{ Europe occidentale } \\
\hline Allemagne & $\sim 85 \%$ & $46132-54565$ & $23-28$ & $1,1-1,3$ & 1,36 & - & 2361 \\
\hline Belgique & $66 \%$ & $9847-14985$ & $40-61$ & $1,7-2,7$ & 1,60 & 15,2 & 2050 \\
\hline France & $100 \%$ & 46800 & 32 & 1,4 & 1,75 & 8,3 & 2043 \\
\hline Pays-Bas & $100 \%$ & 13865 & 35 & 1,4 & 1,63 & 16,9 & 2150 \\
\hline Suisse & $100 \%$ & 4002 & 23 & 1,0 & 1,47 & - & 2853 \\
\hline
\end{tabular}




\begin{tabular}{|c|c|c|c|c|c|c|c|}
\hline Pays & $\begin{array}{l}\% \text { de centres } \\
\text { enregistrés }\end{array}$ & $\begin{array}{c}\text { Nombre } \\
\text { de cycles (a) }\end{array}$ & $\mid \begin{array}{l}\text { Nombre de cycles } \\
\text { p. } 10000 \text { femmes } \\
\text { de } 15-49 \text { ans }\end{array}$ & $\begin{array}{l}\text { \% d'enfants nés } \\
\text { par fécondation } \\
\text { assistée(b) }\end{array}$ & $\begin{array}{c}\text { Indice synthé- } \\
\text { tique de fécondité } \\
\text { (enfants } \\
\text { par femme) }\end{array}$ & $\begin{array}{l}\text { Taux d'infécon- } \\
\text { dité définitive } \\
\text { dans la généra- } \\
\text { tion } 1955\end{array}$ & $\begin{array}{l}\text { Dépenses de } \\
\text { santé par habi- } \\
\text { tant en } 1998 \\
(\mathrm{SPA})^{(\mathrm{c})}\end{array}$ \\
\hline \multicolumn{8}{|c|}{ Europe septentrionale } \\
\hline Danemark & $100 \%$ & 8409 & 66 & 3,0 & 1,72 & 12,9 & 2132 \\
\hline Finlande & $100 \%$ & 7547 & 31 & 2,6 & 1,70 & 15,5 & 1510 \\
\hline Islande & $100 \%$ & 383 & 54 & 3,9 & 2,04 & - & 2113 \\
\hline Norvège & $100 \%$ & 3643 & 34 & 1,7 & 1,81 & 13,5 & 2452 \\
\hline Royaume-Uni & $100 \%$ & 33647 & 24 & 1,1 & 1,71 & - & 1510 \\
\hline Suède & $100 \%$ & 8381 & 42 & 2,4 & 1,50 & 12,8 & 1732 \\
\hline \multicolumn{8}{|c|}{$\begin{array}{l}\text { (a) Il s'agit du nombre total de cycles réalisés dans le cadre de FIV, d'ICSI et de TEC. Les fourchettes sont des estimations : la borne inférieure est le nombre de cycles } \\
\text { déclarés dans le bilan, la borne supérieure est le nombre de cycles estimé en supposant que les centres non enregistrés ont une activité identique à celle des centres enre- } \\
\text { gistrés. } \\
\text { (b) Le nombre estimé d'enfants nés par FIV, ICSI ou TEC suite à un cycle réalisé durant l'année } 1998 \text { a été rapporté au nombre d'enfants nés dans le pays au cours de } \\
\text { l'année } 1999 \text {. Le nombre d'enfants nés par FIV, ICSI ou TEC a été estimé à partir des données sur le nombre d'accouchements et sur la distribution des grossesses mul- } \\
\text { tiples (données rapportées dans le bilan européen). L'information sur le devenir des grossesses était manquante pour les Pays-Bas et la Belgique et de très mauvaise qua- } \\
\text { lité pour la Suisse. Pour ces trois pays, le nombre d'enfants nés par FIV, ICSI ou TEC a été estimé en supposant que ces pays ont des taux de succès identiques aux taux } \\
\text { de succès moyens des } 15 \text { autres pays. Cette hypothèse a également été appliquée à l'Espagne pour estimer le nombre d'enfants nés par TEC car ce pays manquait (sans } \\
\text { explication) dans une partie des tableaux du bilan européen. Par ailleurs, le bilan européen présentait des incohérences pour les données du Royaume-Uni et des hypo- } \\
\text { thèses de cohérence ont été faites pour ce pays. } \\
\text { (c) Les SPA (standards de pouvoir d'achat) sont une unité indépendante des monnaies nationales. La conversion des dépenses en SPA permet d'effectuer des comparai- } \\
\text { sons en volume. } \\
\text { Sources: Bilan européen de fécondation assistée } 1998 \text { (Nygren et Andersen, 2001b), Observatoire démographique européen, annuaire Eurostat } 2002 \text { et bilan } \\
\text { démographique des pays développés (Sardon, 2002). }\end{array}$} \\
\hline
\end{tabular}


Les variations du recours à la FIV entre pays européens conduisent à s'interroger sur les raisons de telles différences. Une première possibilité est de tenter d'expliquer « simplement» les variations du recours à la FIV à partir des caractéristiques du pays. Dans cette optique, on pourrait penser que le développement de cette pratique est corrélé à la dynamique de fécondité du pays, les populations désirant avoir des enfants étant peutêtre plus susceptibles d'y recourir. La figure $1 \mathrm{~b}$ représente les variations du recours à la FIV en fonction de l'indice synthétique de fécondité (ISF) : le lien apparaît ténu. On peut également envisager que le recours à la FIV soit un reflet des problèmes de stérilité rencontrés par les populations, mais on ne dispose pas d'indicateur permettant de mesurer directement ce niveau de stérilité(11). Dans les générations anciennes, le taux d'infécondité définitive était sans doute fortement lié au niveau d'infécondité involontaire. Ce taux, qui était relativement stable jusqu'aux générations nées en 1960, a augmenté fortement dans les générations suivantes, reflétant désormais non seulement l'infécondité involontaire mais aussi l'infécondité volontaire (Sardon, 2002). Le tableau 3 indique le taux d'infécondité définitive de la génération 1955, dont nous supposons qu'il est fortement lié au niveau d'infécondité involontaire. Là encore, aucun lien n'apparaît nettement entre ce taux et la fréquence du recours à la FIV (figure 1c). D'autres explications peuvent encore être envisagées, en particulier de type économique, comme le niveau des dépenses de santé (tableau 3) : cette piste ne semble pas plus pertinente que les précédentes (figure 1d). Ces premiers éléments ne permettent donc pas d'avancer dans la compréhension des variations du recours à la FIV en Europe. Pour progresser dans ce domaine, il serait intéressant d'étudier les conditions de la prise en charge de l'infertilité dans les pays européens, en examinant par exemple le coût économique des FIV pour les couples (en fonction de leur prise en charge ou non par les régimes de sécurité sociale qui varie beaucoup selon les pays(12)), le niveau de l'offre dans le domaine de la fécondation in vitro (niveau de formation des professionnels de santé assurant le suivi gynécologique des femmes, nombre, taille et répartition des centres de FIV sur le territoire) ou encore en explorant l'acceptation de ces méthodes par les populations. Le coût financier supporté par les couples pourrait être un facteur déterminant : ainsi, au Royaume-Uni, la décision de tenter une deuxième FIV après un premier échec semble déterminée non seulement par la perception de son pronostic de succès (cette

(11) Pour les démographes, la notion de stérilité s'oppose à celle de fertilité, qui désigne l'aptitude à concevoir et à mener à son terme normal une grossesse (Leridon, 1981). Pour mesurer le niveau de stérilité d'une population, les démographes ont proposé d'utiliser un indicateur indirect : le taux d'infécondité involontaire, c'est-à-dire le pourcentage de femmes mariées restant involontairement sans enfant.

(12) Dans un article analysant les implications économiques des techniques d'assistance médicale à la reproduction (Garceau et al., 2002), les auteurs signalent par exemple que les FIV sont complètement remboursées en France, qu'elles le sont partiellement en Belgique, au Danemark et en Norvège. Le Royaume-Uni se caractérise par une forte variation régionale de cette prise en charge, qui se traduirait au niveau national par la prise en charge d'une FIV sur quatre seulement. 

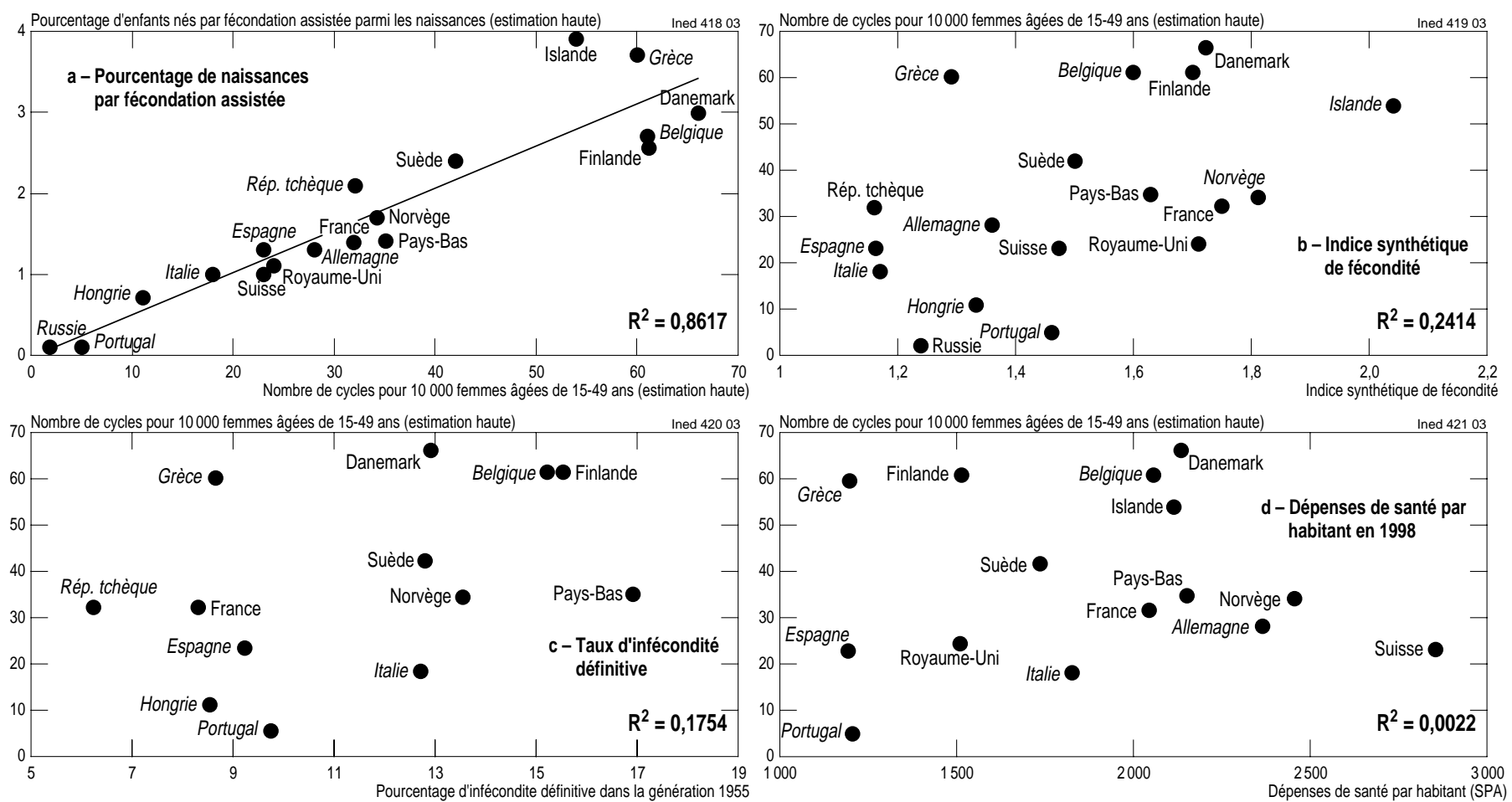

Figure 1.- Recours à la fécondation assistée (mesurée par le nombre de cycles pour 10000 femmes âgées de 15-49 ans) et indicateurs démographiques et économiques

Note : en italique figurent les pays dont l'activité FIV n'est pas connue de façon exhaustive et repose sur une estimation haute (cf. tableau 3).

Sources : Bilan européen de fécondation assistée 1998 (Nygren et Andersen, 2001b), Observatoire démographique européen, annuaire Eurostat 2002 et bilan démographique des pays développés (Sardon, 2002). 
perception s'appuyant sur des facteurs tels que l'âge de la femme, le nombre d'ovocytes ponctionnés et le nombre d'embryons transférés durant la première tentative de FIV) mais aussi par la capacité du couple à financer la suite du traitement dans un contexte national où la prise en charge sociale du coût des FIV est très limitée et variable (Sharma et al., 2002).

\section{La FIV : une technique inadaptée au traitement des infertilités masculines sévères}

\section{Comment mesurer le succès des FIV?}

Dans la littérature, la mesure du taux de succès des FIV est généralement basée sur le pourcentage de tentatives ayant permis la réalisation d'une ponction folliculaire se terminant par une grossesse clinique, dit taux de grossesses par ponction. À partir de FIVNAT, on estime que ce taux de succès est de $20 \%$ à $25 \%$. Ainsi formulée, la mesure des taux de succès peut sembler simple; en réalité, elle soulève de nombreuses difficultés qui ont donné lieu à des polémiques (Marcus-Steiff, 1990 et 1991 ; Thibault et al., 1991; Humeau et Arnal, 1994 ; Marcus-Steiff, 1994a et 1994b; Tain, 2003b). Ce taux de grossesses par ponction pose plus spécifiquement quatre questions.

Premièrement, la population admise en FIV inclut à la fois des couples stériles, c'est-à-dire des couples ayant une probabilité nulle de concevoir sans aide médicale, et des couples hypofertiles, qui ont une probabilité non nulle de concevoir « naturellement» (Leridon, 1981). Pour mesurer l'efficacité réelle de la FIV, il serait nécessaire de prendre en compte l'existence de ceux que l'on a appelés les «faux bébés FIV » (Marcus-Steiff, 1994a), c'est-à-dire les bébés conçus naturellement pendant le cycle de FIV et les bébés qui auraient pu être conçus naturellement au cours de ce cycle si l'on n'avait pas fait de FIV. Autrement dit, l'évaluation des taux de succès devrait prendre en compte les probabilités de conception hors traitement médical des couples hypofertiles (Leridon et Spira, 1984 ; Leridon, 1990).

Deuxièmement, le taux de grossesses par ponction ne permet pas de connaître la proportion de couples obtenant la naissance d'un enfant vivant, puisqu'une partie des grossesses cliniques se termine par un décès fœtal (fausse-couche, grossesse extra-utérine, enfant mort-né). Pour avoir une mesure globale du succès des FIV, il faut estimer le taux de tentatives menant à la naissance d'au moins un enfant vivant parmi les tentatives ayant permis la réalisation d'une ponction folliculaire. Ce taux est appelé en anglais le take home baby rate (taux de bébés rentrant à la maison) et il atteindrait $15 \%$ à $20 \%$ des ponctions, sur la base des données FIVNAT $^{(13)}$. Ce taux peut être estimé en première approximation par le 
taux d'accouchements (en négligeant la mortalité néonatale). Au-delà du taux de bébés rentrant à la maison, un groupe d'experts européens a souligné qu'il serait nécessaire que les comités de rédaction des journaux scientifiques imposent l'évaluation des succès en termes de survenue d'une grossesse unique menant à la naissance d'un enfant en bonne santé (ESHRE Campus Course Report, 2001). Cette discussion autour des indicateurs de succès (obtenir la naissance d'au moins un enfant ou obtenir une grossesse unique menant à la naissance d'un enfant en bonne santé) renvoie plus largement à des débats autour des «bonnes pratiques cliniques », recommandant de prévenir les grossesses multiples en limitant le nombre d'embryons transférés durant un cycle de FIV (Lambert, 2002).

Troisièmement, pour déterminer les succès réels de la FIV, il faut mesurer la probabilité de succès en termes de parcours de FIV (pouvant comprendre plusieurs tentatives) et non pas uniquement en termes de cycles de FIV. Une telle approche a été récemment mise en œuvre dans un service anglais à partir de 2056 couples débutant un cycle de FIV (Sharma et al., 2002). Ces couples ont été suivis jusqu'à ce qu'ils obtiennent une grossesse $(n=879)$, qu'ils abandonnent le programme de FIV $(n=1155)$ ou qu'ils aient réalisé quatre tentatives sans succès $(n=22)$. À partir de cette cohorte, les auteurs estiment qu'au bout de quatre tentatives, $75 \%$ des couples ont obtenu au moins une fois une grossesse clinique et que $66 \%$ ont obtenu la naissance d'au moins un enfant vivant. Cependant, beaucoup de couples abandonnent avant ces quatre tentatives : après chaque tentative de FIV, plus de $60 \%$ des couples n'ayant pas obtenu de grossesse décident d'arrêter. Au niveau de la cohorte observée, $56 \%$ des couples ont décidé d'arrêter le programme de FIV sans avoir obtenu de grossesse, $1 \%$ des couples ont poursuivi leurs tentatives jusqu'à quatre FIV sans succès, et $43 \%$ ont obtenu une grossesse qui s'est terminée dans plus de $80 \%$ des cas par une naissance vivante.

Quatrièmement, les taux de succès varient de manière importante selon les caractéristiques du couple; on observe en particulier un effet très important de l'âge de la femme et des caractéristiques spermatiques de l'homme (Hull et al., 1992). Nous allons développer ce point.

\section{Chute des taux de succès avec l'âge de la femme}

La figure 2 présente les taux de grossesses par ponction et les taux d'accouchements par ponction en fonction de l'âge de la femme à partir des données FIVNAT pour la période 1987-1996 (FIVNAT et al., 1990 ; FIVNAT et al., 1991; FIVNAT et al., 1997). On observe une baisse importante des taux de succès après 35-37 ans : le taux de tentatives menant à un accouchement passe de $16,4 \%$ à 30 ans à $9,3 \%$ à 40 ans et $2,8 \%$ à

(13) Si l'on prend en compte les transferts d'embryons congelés, ce taux de succès augmente de 2 à 3 points. 
45 ans. Du coup, la prise en charge des femmes âgées de plus de 35-37 ans est faite dans l'urgence afin d'accélérer la procédure. Ainsi, le délai moyen séparant la première consultation de la première FIV passe de plus d'une année chez les femmes âgées de moins de 35 ans à 6 mois chez les femmes âgées de 35 ans ou plus, d'après une étude réalisée dans un service hospitalier de gynécologie clinique sur une cohorte de femmes $(n=340)$ ayant consulté une première fois en 1987 ou 1991 (Tain, 2001).

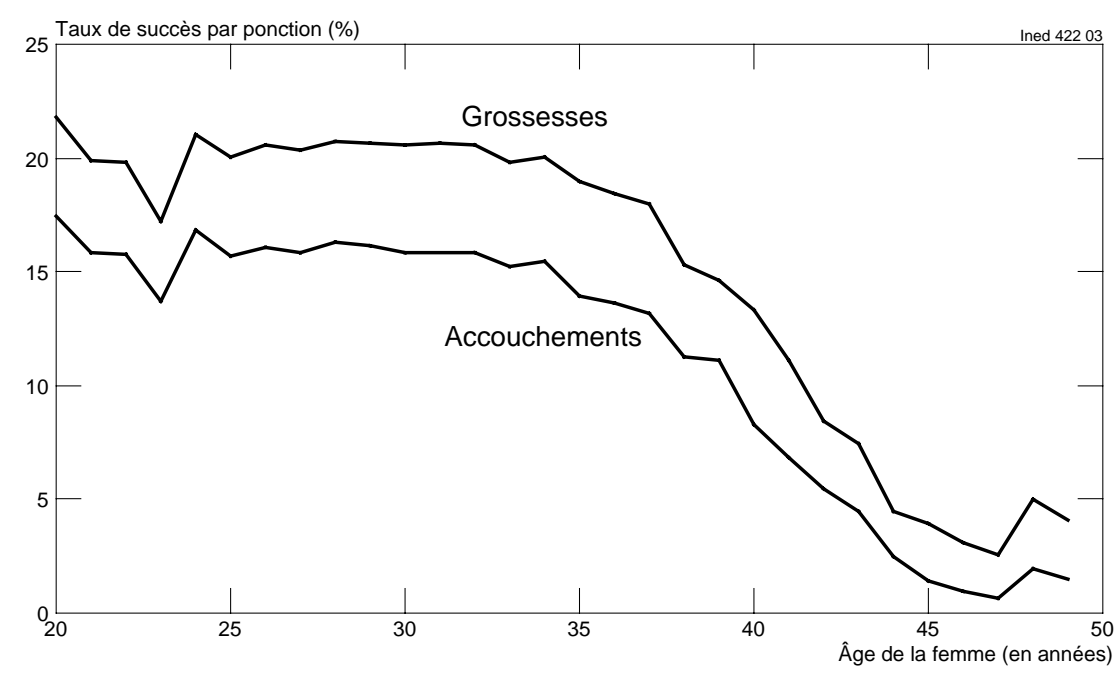

Figure 2.- Évolution du taux de succès des FIV en fonction de l'âge de la femme de 1987 à 1996 (en \%)

Source : données issues de FIVNAT (Contraception, Fertilité, Sexualité, 1997, 25(7-8), p. 503-506, tableau 2).

\section{Chute des taux de succès lorsque l'homme a un sperme sévèrement altéré}

Le taux de succès varie également en fonction des caractéristiques du sperme. Initialement, la FIV a été mise au point pour répondre aux infertilités féminines liées à des anomalies des trompes de Fallope, mais le recours à la FIV a été étendu aux autres indications féminines et à des indications masculines et idiopathiques. Les indications masculines sont définies par une anomalie du sperme, en général un spermogramme avec une numération inférieure à 20 millions de spermatozoïdes par millilitre et/ou un pourcentage de spermatozoïdes mobiles inférieur à $40 \%$ et/ou un pourcentage de spermatozoïdes morphologiquement normaux inférieur à $40 \%$ (Plachot, 1987). Avec cette définition, on observe sur la base des données FIVNAT que la proportion de FIV réalisées en raison d'une indication 
masculine pure (c'est-à-dire chez des couples dans lesquels la femme présente une fertilité normale) a progressé de $11 \%$ à $22 \%$ entre 1986 et 1992 ; en parallèle, la proportion de FIV liées à une indication féminine pure (en général une anomalie des trompes de Fallope) a chuté de $60 \%$ à $44 \%$. Cette progression des indications masculines reste cependant limitée par les faibles taux de succès des FIV dans le cas d'infertilité masculine sévère. Ces échecs sont liés à une diminution des chances de fécondation in vitro tandis que les chances de développement des embryons obtenus ne semblent pas altérées (Giorgetti, 1987; Tournaye et al., 1992). Ainsi, le taux de fécondation (nombre d'embryons obtenus rapporté au nombre d'ovocytes mis en fécondation) passe de $72 \%$ à $18 \%$ lorsque le sperme est sévèrement altéré, c'est-à-dire lorsque le pourcentage de spermatozoïdes morphologiquement normaux est inférieur à $5 \%$ et que le nombre de spermatozoïdes mobiles par millilitre est inférieur à 3 millions (Enginsu et al., 1992).

Devant cette chute des taux de succès, le Guide des bonnes pratiques cliniques et biologiques en Assistance Médicale à la Procréation (CNMBRDP, 1999) indique que la technique de FIV doit être réservée aux hommes ayant au minimum 50000 spermatozoïdes mobiles par millilitre de sperme. Quelles sont alors les alternatives en cas d'infertilité masculine sévère?

\section{L'IAD : une technique mal acceptée}

En cas d'infertilité masculine sévère, la médecine a d'abord proposé l'insémination artificielle avec spermatozoïdes de donneur. L'insémination artificielle (IA) consiste à déposer du sperme dans les voies génitales féminines (cul-de-sac vaginal, col de l'utérus, cavité utérine). Le sperme inséminé peut être celui du conjoint (IAC) ou celui d'un donneur (IAD). Parmi les couples ayant recours à l'IAD, on distingue quatre indications : les azoospermies (absence totale de spermatozoïdes) dans $53 \%$ des cas, les oligospermies (moins de 20 millions de spermatozoïdes par millilitre) dans $38 \%$ des cas, les asthéno-tératospermies (diminution du pourcentage de spermatozoïdes mobiles et/ou du pourcentage de spermatozoïdes morphologiquement normaux) dans $7 \%$ des cas, et les IAD réalisées pour des raisons génétiques ${ }^{(14)}$ dans $2 \%$ des cas ${ }^{(15)}$.

(14) L'IAD pour cause génétique permet d'éviter de transmettre à l'enfant une maladie génétique engageant son pronostic vital ou entraînant un handicap sévère (maladie dominante chez le père par exemple). Cf. le Guide des bonnes pratiques cliniques et biologiques en Assistance Médicale à la Procréation (CNMBRDP, 1999).

(15) Ces pourcentages correspondent à la distribution des indications d'IAD réalisées en France pour la période 1984-1987 (Fédération française des CECOS et al., 1989). 


\section{Des règles déontologiques communes à l'ensemble des centres français}

L'IAD a déjà une longue histoire derrière elle : la première IAD réussie a été réalisée aux États-Unis en 1884. Dès 1953, des naissances sont obtenues à partir de spermatozoïdes congelés (Netter et Belaisch, 1991). À partir de ces avancées technologiques, la France met en place en 1973, sous l'impulsion du professeur Georges David, des centres pour assurer la congélation et la conservation des spermatozoïdes, appelés Centres d'étude et de conservation du sperme humain (CECOS). Par la suite, les CECOS étendront leur activité à la congélation et à la conservation des œufs et des embryons(16) : le sigle CECOS signifie désormais Centre d'étude et de conservation des œufs et du sperme humain. Le fonctionnement français des «banques de sperme » est unique en raison du regroupement de la vingtaine de CECOS au sein de la Fédération des $\operatorname{CECOS}^{(17)}$. Cette fédération permet à la fois une homogénéisation des pratiques dans l'ensemble des centres et la publication de bilans nationaux d'IAD. Au niveau des pratiques, le système français est basé sur des règles de déontologie communes établies initialement par le professeur George David(18) : le donneur doit être volontaire, père de famille, et avoir l'accord de sa conjointe; de plus, son don est gratuit et anonyme (MadaniPerret, 1987).

\section{Les chiffres de l'IAD en France}

La Fédération des CECOS collecte les données de l'IAD et les publie annuellement ${ }^{(19)}$; un bilan «consolidé » pour les quinze premières années d'exercice des CECOS (période 1973-1987) a été publié en 1989 (Fédération française des CECOS et al., 1989). Les huit premières années (1973-1980) ont été marquées par une forte progression du nombre de couples déposant une première demande, suivie d'une période de stabilisation autour de 3000 nouvelles demandes par an. Les données relatives aux IAD de la période 1986-1998 sont présentées dans le tableau 4. Durant les années 1990-1992, 20000 cycles d'IAD ont été réalisés chaque année,

(16) L'assistance médicale à la procréation avec don de gamètes comprend désormais l'IAD et la FIV avec tiers donneur. Dans le cadre de la FIV avec tiers donneur, il peut s'agir d'un don de spermatozoïdes ou d'un don d'ovocytes, le double don étant interdit (article L. 152-3, alinéa 1 du Code de la Santé publique). Lors d'un cycle de FIV, si le nombre d'embryons obtenus est supérieur au nombre d'embryons transférés, le couple a la possibilité de demander la congélation des embryons non transférés en vue de pratiquer un transfert d'embryon congelé (TEC) lors d'une tentative ultérieure.

(17) La Fédération des CECOS dispose d'un site Internet à l'adresse suivante : http://amp-chu-besancon.univ-fcomte.fr/cecos/default.htm

(18) Depuis 1994, l'activité d'IAD est encadrée par la loi de bioéthique.

(19) Jusqu'en 1999, le bilan de la Fédération des CECOS était publié dans Contraception, Fertilité, Sexualité. Depuis l'année 2000, il est publié dans Reproduction Humaine et Hormones. Les données de l'IAD sont également collectées dans le cadre des bilans d'activité réalisés pour le ministère de la Santé. 
donnant lieu annuellement à quelque 1400 accouchements et à la naissance de 1500 enfants.

Contrairement au système de collecte mis en place pour la FIV, on ne dispose pas de bilans mondiaux ni européens concernant l'IAD. La publication de bilans nationaux sur l'IAD est en effet une spécificité française, liée au fonctionnent fédératif des «banques de sperme » dans notre pays (Fédération française des CECOS et al., 1989). Sans données équivalentes aux niveaux européen et mondial, on manque de matière pour effectuer des analyses comparatives sur cette technique.

TABLEAU 4.- BILAN DES PROCRÉATIONS MÉDICALEMENT ASSISTÉES AVEC DON DE SPERMATOZOÏDES EN FRANCE DE 1986 À 1998 (ESTIMATIONS)

\begin{tabular}{|l|c|c|c|c|c|c|}
\hline \multirow{2}{*}{ Année } & \multirow{2}{*}{$\begin{array}{c}\text { Centres } \\
\text { CECOS }^{(a)}\end{array}$} & \multicolumn{2}{|c|}{ Cycles $^{(\mathrm{b} ; \mathrm{c})}$} & $\begin{array}{c}\text { Grossesses } \\
\text { cliniques }\end{array}$ & $\begin{array}{c}\text { Accouche- } \\
\text { ments }(\mathrm{d})\end{array}$ & Enfants(d) \\
\cline { 3 - 6 } 1986 & 20 & 23926 & - & 1938 & 1550 & 1674 \\
1987 & 20 & 23192 & 1408 & 2204 & 1746 & 1939 \\
1988 & 20 & 21446 & 1864 & 2232 & 1762 & 1976 \\
1989 & 20 & 20004 & 2151 & 2186 & 1720 & 1947 \\
1990 & 20 & 20958 & 2329 & 2377 & 1869 & 2095 \\
1991 & 22 & 20190 & 2313 & 2227 & 1746 & 1981 \\
1992 & 22 & 18260 & 2201 & 2219 & 1757 & 2004 \\
1993 & 22 & 17723 & 2146 & 2167 & 1672 & 1908 \\
1994 & 22 & 16887 & 1921 & 2048 & 1610 & 1860 \\
1995 & 22 & 12510 & 1674 & 1620 & 1309 & 1501 \\
1996 & 22 & 10539 & 1254 & 1359 & 1005 & 1165 \\
1997 & 22 & 9735 & 1182 & 1321 & 937 & 1082 \\
1998 & 23 & 8145 & 1294 & 1351 & 859 & 1001 \\
\hline Total(e) & & 223515 & 21737 & 25249 & 19542 & 22133 \\
\hline
\end{tabular}

(a) Les CECOS (Centres d'étude et de conservation des œufs et du sperme humain) ont été créés en 1973. Au nombre d'une vingtaine, ils sont regroupés au sein de la Fédération des CECOS qui coordonne et harmonise le fonctionnement des centres, réalise et publie le bilan annuel de leurs activités.

(b) Les cycles sont divisés en IAD et en FIV-D. L'IAD (insémination artificielle avec spermatozoïdes de donneur) regroupe les inséminations intracervicales et les inséminations intra-utérines. La FIV-D (fécondation in vitro avec spermatozoïdes de donneur) regroupe les FIV classiques, les GIFT (Gamete Intra Fallopian Transfer, transfert intra-tubaire de gamètes) et les ICSI (IntraCytoplasmic Sperm Injection, injection intra-cytoplasmique de spermatozoïde).

(c) Les nombres de cycles (d'IAD et de FIV-D) sont issus des bilans d'activité des centres CECOS publiés annuellement par la Fédération des CECOS dans le numéro 7-8 de la revue Contraception, Fertilité, Sexualité entre 1987 et 1999. Pour les années 1986-1991, les données portent uniquement sur les centres CECOS. À partir de 1992, le bilan incorpore également l'activité des centres privés agréés, c'est-à-dire essentiellement l'IFREARES (Institut francophone de recherche et d'études appliquées à la reproduction et à la sexologie) de Toulouse qui représente $1 \%$ à $1,5 \%$ de l'activité d'assistance médicale à la procréation avec don de spermatozoïdes.

(d) Les nombres d'accouchements et d'enfants sont estimés à partir de l'hypothèse d'un taux de succès identique pour les grossesses perdues de vue et pour les grossesses enregistrées dans le bilan. L'information sur les accouchements et les enfants n'est disponible qu'à partir de 1990. Pour la période 1986-1989, le nombre d'accouchements et d'enfants est estimé en supposant que les taux de succès sur cette période sont identiques à ceux observés sur la période 1990-1991.

(e) Pour la période 1986-1998, les 223515 cycles d'IAD ont abouti à 19976 grossesses, 15617 accouchements, 16916 enfants. Les 21737 cycles de FIV-D ont abouti à 5273 grossesses, 3925 accouchements, 5217 enfants.

Sources : voir notes ci-dessus. 


\section{De la difficulté d'accepter un don lorsqu'il s'agit de spermatozoüdes}

Une enquête réalisée par l'Ined à la fin de l'année 1976 avait permis de mesurer la connaissance que la population française avait de la technique d'IAD à cette période. Trois années après la mise en place des CECOS, $79 \%$ des personnes interrogées déclaraient avoir déjà entendu parler de l'IAD $^{(20)}, 19 \%$ n'en avoir jamais entendu parler et $2 \%$ ne se prononçaient pas (Leridon, 1980). Une dizaine d'années plus tard, la quasi-totalité $(96 \%)$ du public avait entendu parler de la technique d'insémination artificielle (Charbit, 1989). Mais cette connaissance répandue de l'IAD ne signifie pas forcément que cette technique soit bien acceptée.

L'IAD soulève en effet la question de l'acceptation ou non du principe d'un «donneur ». Deux enquêtes réalisées par l'Ined à dix-huit ans d'intervalle, en 1976 et en 1994, permettent de connaître l'opinion des Français sur cette question. Dans l'enquête de 1976, la question posée était la suivante : "Pensez-vous que ce soit une bonne solution [le recours à l'IAD] pour un ménage qui voudrait absolument avoir un enfant et ne pourrait pas en avoir autrement? ». Parmi les personnes qui avaient déjà entendu parler de l'IAD, $49 \%$ ont répondu «non », $41 \%$ « oui » et $10 \%$ se sont abstenues (Leridon, 1980). Les personnes opposées à l'IAD étaient interrogées sur les raisons de ce refus; trois raisons totalisent $61 \%$ des réponses : «Il est plus simple d'adopter un enfant » $(25 \%)$, «L'enfant serait de la mère seulement, pas du mari » $(23 \%)$, «Le donneur pourrait transmettre des tares » $(13 \%)$. Ces chiffres indiquaient donc une méfiance de l'opinion par rapport à l'IAD. Cependant, cette méfiance pouvait s'expliquer par la création encore récente des CECOS au moment de l'enquête (moins de quatre années) et, en 1980, lors de la publication de ces chiffres, Henri Leridon concluait :

«Insistons sur le fait que l'enquête est "vieille" de trois ans; or dans des domaines comme celui-ci, l'opinion évolue très vite, et il y a tout lieu de penser qu'aujourd'hui elle se montrerait nettement plus "libérale" qu'il y a deux ou trois ans. » (p. 158)

Qu'en était-il dix-huit ans plus tard, en 1994, lors de l'enquête Situations familiales et emploi (cf. note 1)? Les personnes enquêtées y ont été interrogées sur les méthodes d'assistance médicale à la procréation, l'IAD et la FIV ${ }^{(21)}$. Après qu'on leur ait expliqué ces deux techniques(22), les en-

(20) Après avoir interrogé le couple sur la solution de l'adoption pour des couples stériles, l'IAD était évoquée en ces termes : «Dans le cas où c'est le mari qui ne peut pas avoir d'enfant, il est également possible à la femme d'avoir un enfant en recourant à l'insémination artificielle (fécondation sans relation sexuelle). Aujourd'hui, cela peut être fait avec la semence d'un autre homme, qui reste inconnu du ménage, qui n'est pas rémunéré, qui le fait avec l'accord de sa femme, et sous contrôle médical. Avez-vous entendu parler de cette méthode? ».

(21) Données non publiées.

(22) La FIV était décrite ainsi : «En laboratoire on féconde l'ovule de la femme avec le sperme du mari, l'embryon est ensuite replacé dans le ventre de la mère »; l'IAD était présentée de la façon suivante : «la femme est fécondée par le sperme d'un autre homme que son mari, après un don de sperme anonyme ». 
quêtés devaient déclarer si, pour un couple ne pouvant pas avoir d'enfant, il valait mieux : 1) avoir recours à la FIV ou adopter un enfant ?;2) avoir recours à une IAD ou adopter un enfant? Les réponses sont présentées dans le tableau 5 selon le sexe de l'enquêté et selon qu'il a déjà eu luimême des difficultés pour avoir un enfant ou non. Quel que soit le statut du répondant, la FIV est préférée à l'adoption (49\% à $68 \%$ en faveur de la FIV, contre $24 \%$ à $35 \%$ en faveur de l'adoption) mais l'adoption est préférée à l'IAD (49\% à $55 \%$ en faveur de l'adoption, contre $24 \%$ à $39 \%$ en faveur de l'IAD). Ces réponses mettent en évidence une attitude de la population française très différente devant ces deux techniques : en comparant ces déclarations, on peut conclure que parmi les techniques d'assistance médicale à la procréation, l'IAD est une technique mal acceptée. Malgré la différence de formulation entre les enquêtes de 1976 et de 1994, ces chiffres indiquent que la population française reste réticente devant l'IAD, probablement en raison de la difficulté à accepter l'idée d'un don de sperme.

TABLEAU 5.- ACCEPTATION DE LA FIV ET DE L'IAD EN FRANCE EN 1994 SELON LE SEXE ET L'EXISTENCE OU NON DE DIFFICULTÉS POUR AVOIR UN ENFANT (\%)

\begin{tabular}{|c|c|c|c|c|}
\hline $\begin{array}{l}\text { Ce tableau présente les re } \\
\text { 1) Pour un couple qui n } \\
\text { supposant que la deman } \\
\text { 2) Pour un couple qui n } \\
\text { supposant que la deman }\end{array}$ & $\begin{array}{l}\text { ses à deux q } \\
\text { ut avoir d'er } \\
\text { d'adoption a } \\
\text { ut avoir d'er } \\
\text { d'adoption a }\end{array}$ & $\begin{array}{l}\text { mieux recou } \\
\text { t mieux recou }\end{array}$ & he FIV c & $\begin{array}{l}\text { enfant (en } \\
\text { enfant (en }\end{array}$ \\
\hline Femmes & $\begin{array}{r}\text { N'ayant ja } \\
\text { pour }\end{array}$ & $\begin{array}{l}\text { e difficultés } \\
\text { enfant }\end{array}$ & $\begin{array}{r}\text { Ayant } \\
\text { pour }\end{array}$ & $\begin{array}{l}\text { icultés } \\
\text { nfant }\end{array}$ \\
\hline & FIV & IAD & FIV & IAD \\
\hline $\begin{array}{l}\text { Recourir à la méthode } \\
\text { Adopter un enfant } \\
\text { Ne se prononce pas }\end{array}$ & $\begin{array}{r}57,5 \\
31,6 \\
10,9 \\
100,0\end{array}$ & $\begin{array}{r}31,7 \\
53,3 \\
15,0 \\
100,0\end{array}$ & $\begin{array}{r}66,0 \\
25,4 \\
8,6 \\
100,0\end{array}$ & $\begin{array}{r}38,5 \\
49,1 \\
12,4 \\
100,0\end{array}$ \\
\hline Hommes & $\begin{array}{r}\text { N'ayant ja } \\
\text { pour }\end{array}$ & $\begin{array}{l}\text { e difficultés } \\
\text { nfant }\end{array}$ & $\begin{array}{r}\text { Ayant } \\
\text { pour }\end{array}$ & $\begin{array}{l}\text { icultés } \\
\text { nfant }\end{array}$ \\
\hline & FIV & IAD & FIV & IAD \\
\hline $\begin{array}{l}\text { Recourir à la méthode } \\
\text { Adopter un enfant } \\
\text { Ne se prononce pas }\end{array}$ & $\begin{array}{r}49,1 \\
34,3 \\
16,6 \\
100,0\end{array}$ & $\begin{array}{r}24,6 \\
54,7 \\
20,7 \\
100,0\end{array}$ & $\begin{array}{r}67,8 \\
24,0 \\
8,2 \\
100,0\end{array}$ & $\begin{array}{r}32,0 \\
52,2 \\
15,8 \\
100,0\end{array}$ \\
\hline
\end{tabular}

Une enquête sociodémographique réalisée auprès de 1041 couples ayant déposé une demande d'IAD au cours de l'année 1985 aux CECOS du Kremlin-Bicêtre (Île-de-France) et de Rennes (Bretagne) (Levy, 1994) permet de mieux cerner ces difficultés. Dans cette population, $43 \%$ des 
couples ont abandonné la procédure d'IAD. Les raisons de ce fort taux d'abandon sont difficiles à établir car dans $57 \%$ des cas, les couples ont coupé tout contact avec le CECOS sans donner d'explication.

Qu'en est-il dans les autres pays? Dans ce domaine, la législation diffère d'un pays à l'autre, puisque certains pays ont interdit ou restreint le recours au don de sperme (Hamberger et Janson, 1997). Dans les pays autorisant le don de sperme, des réticences par rapport à cette méthode ont en outre été relevées parmi les couples inféconds qui sont confrontés à une attitude négative de la société(23) face à cette méthode (Mahlstedt et Greenfeld, 1989; Braverman et Corson, 1995). L'attitude de l'entourage familial pourrait avoir un impact tout particulier, les couples inféconds s'interrogeant plus spécifiquement sur le lien qui unira leur enfant né par IAD à ses grands-parents (Mahlstedt et Greenfeld, 1989).

Si des différences d'attitude existent devant l'IAD, celles-ci semblent plutôt liées au sexe des personnes interrogées. Au sein du couple, la décision d'entamer un programme d'IAD pourrait être très majoritairement prise par les femmes (Rojo-Moreno et al., 1994 ; Rojo-Moreno et al., 1996)(24). On note surtout une attitude des hommes et des femmes très différente face à la question du secret : les hommes désirent plus souvent garder le secret sur l'existence d'un donneur que les femmes (Braverman et Corson, 1995). On a supposé qu'en gardant le secret, les hommes cherchaient à protéger leur relation avec l'enfant, basée sur un lien non génétique. Cependant, cette hypothèse est peut-être trop simpliste : on observe également une différence d'attitude des hommes et des femmes à propos des FIV avec don d'ovocytes, puisque les hommes désirent, plus souvent que leur partenaire, garder le secret sur le recours à une donneuse (Braverman et Corson, 1995).

Devant ces difficultés, il est apparu nécessaire aux équipes médicales de donner du «temps » aux patients avant de commencer une procédure d'IAD (Mahlstedt et Greenfeld, 1989). Par exemple, l'équipe du CECOS de Midi-Pyrénées (Hôpital de la Grave, Toulouse) estime qu'après le diagnostic de stérilité masculine, il est nécessaire d'attendre au moins une année avant d'entamer une IAD, afin de laisser à l'homme le « temps d'élaboration du deuil de la fertilité » (Mazzone, 2000).

(23) Cette attitude négative de la société a été résumée en reprenant la célèbre formule du commandant américain Joshiah Tatnall (1859) : «Blood is thicker than water » (« Le sang est plus épais que l'eau »). On observe en particulier une condamnation de l'IAD par les autorités religieuses, catholiques et juives orthodoxes (Mahlstedt et Greenfeld, 1989). D'après Françoise Héritier-Augé, l'acceptation de «formules » telles que l'IAD ne peut passer que par un soutien sans ambiguïté de la loi, une inscription ferme dans la structure sociale et une adéquation entre l'imaginaire collectif et les représentations de la personne et de 1'identité (Héritier-Augé, 1985).

(24) On ne dispose cependant pas de données similaires pour les autres techniques et l'interprétation de ce résultat est donc difficile. 


\section{L'IAD : un cadre quasi expérimental pour la recherche en reproduction humaine}

Les chercheurs se sont aussi intéressés à l'IAD dans le cadre de l'étude de la reproduction humaine. Les recherches en ce domaine se heurtent en effet à de nombreux problèmes, tels que des biais de sélection, des facteurs de confusion non mesurables, etc. (Baird et al., 1994; Olsen et al., 1998; Weinberg et Dunson, 2000). Face à ces difficultés, les procréations médicalement assistées constituent une voie d'approche originale permettant de contrôler de nombreux paramètres. Cette voie a par exemple été exploitée lors de l'analyse de cycles d'IAD pour étudier l'effet de l'âge maternel sur la fertilité. Dans le cas de l'IAD, les caractéristiques masculines (c'est-à-dire celles du donneur) sont aléatoirement distribuées parmi les femmes : en particulier, l'âge du donneur et l'âge de la femme sont deux paramètres complètement indépendants. Par ailleurs, en sélectionnant des femmes dont le partenaire présente une azoospermie (c'est-à-dire une stérilité totale), on constitue une cohorte de femmes qui n'ont aucune chance de concevoir sans aide médicale et l'on évite les problèmes de biais de sélection généralement rencontrés dans ce type d'étude. Toutes ces caractéristiques font de l'IAD un cadre quasi expérimental pour explorer les effets de l'âge de la femme sur la fertilité humaine. Deux chercheurs français (Schwartz et Mayaux, 1982) ont ainsi mis en évidence un effet de l'âge de la femme qui se traduit par une légère baisse de la fertilité après 30 ans et de manière plus marquée après 35 ans. Ce travail a permis de confirmer les données épidémiologiques et démographiques existantes tout en précisant cet effet de l'âge maternel.

Les données relatives aux FIV pourraient également être exploitées pour étudier des paramètres de la reproduction humaine. En ce qui concerne l'influence de l'âge paternel, l'utilisation de données sur les FIV avec don d'ovocytes ou sur les FIV concernant des couples dans lesquels la femme est totalement stérile (c'est-à-dire présente une stérilité tubaire totale bilatérale) pourrait constituer une piste intéressante.

\section{L'ICSI : l'apparition d'une réponse médicale aux infertilités masculines sévères}

Entre la FIV peu adaptée et l'IAD mal acceptée, la prise en charge de l'infertilité masculine sévère apparaissait donc problématique jusqu'au début des années 1990 (Yovich et Matson, 1995). 


\section{Une nouvelle technique}

Durant l'année 1992, une équipe belge annonce la naissance de quatre bébés (dont une paire de jumeaux) grâce à une nouvelle technique de FIV (Palermo et al., 1992). Classiquement, lors d'une FIV, on met en présence un ovocyte et des spermatozoïdes : ces derniers doivent alors pénétrer l'ovocyte pour le féconder. La nouvelle technique consiste à prendre un spermatozoïde unique et à le faire pénétrer dans un ovocyte à l'aide d'une micro-pipette. Cette technique est nommée ICSI (IntraCytoplasmic Sperm Injection, injection intra-cytoplasmique de spermatozoïde). Dans le cadre de la FIV, on distingue donc désormais les «FIV classiques » ou «FIV conventionnelles » et les ICSI.

L'ICSI permet de traiter les infertilités masculines sévères, avec des spermatozoïdes très peu nombreux, peu mobiles et morphologiquement anormaux (Mansour et al., 1995; Oehninger et al., 1995). Même avec des spermes présentant des anomalies importantes, l'ICSI permet d'obtenir des taux de fécondation (nombre d'embryons obtenus rapporté au nombre d'ovocytes mis en fécondation) élevés, de l'ordre de $71 \%$ (Payne et al., 1994). En 1995, un pas de plus est franchi : Jacques Testart, le «père » d'Amandine, annonce la naissance d'un petit garçon issu de la fécondation d'un ovocyte par une spermatide, c'est-à-dire un spermatozoïde immature (Tesarik et al., 1995). Depuis, la technique de l'ICSI est utilisée pour des hommes présentant une absence de spermatozoïdes dans l'éjaculat et auxquels on prélève, dans le testicule, l'épididyme ou le déférent, des spermatozoïdes et parfois des spermatides ${ }^{(25)}$. D'après l'enquête FIVNAT, parmi les enfants nés par ICSI en 1995-1998, 7 \% ont été conçus avec des spermatozoïdes obtenus par prélèvement chirurgical.

La fécondation in vitro regroupe donc désormais deux grandes techniques, les FIV «classiques », technique développée pour répondre aux infertilités féminines, et les ICSI, technique développée pour répondre aux infertilités masculines sévères. Les infécondités mixtes (féminines et masculines) peuvent être traitées par l'une ou l'autre de ces techniques selon le niveau d'altération du sperme.

\section{Diffusion de l'ICSI}

L'ICSI a été introduite dans le système de collecte des données sur la FIV. Dans l'enquête prospective FIVNAT, 83 tentatives d'ICSI sont enregistrées dès l'année 1992. Le nombre de ponctions déclaré annuellement par les centres englobe, depuis 1992, les ponctions réalisées dans le cadre

(25) Le Guide des bonnes pratiques cliniques et biologiques en Assistance Médicale à la Procréation (CNMBRDP, 1999) précise que dans l'état actuel des connaissances, l'utilisation de spermatides devrait être envisagée uniquement dans le cadre de protocoles de recherche clinique et après avis de la Commission nationale de médecine et de biologie de la reproduction et du diagnostic prénatal (CNMBRDP). 
des ICSI; dans le tableau 2, on observe ainsi une forte progression du nombre total de ponctions réalisées en France, qui est passé de 26000 en 1992 à 41000 en 1999. La figure 3 illustre ce développement à partir de la proportion d'ICSI dans l'ensemble des ponctions enregistrées entre 1992 et 2000 : en sept ans (1994-2000), la part des ICSI est passée de $7 \%$ à $50 \%$. En termes d'effectifs, cela signifie que parmi les 41000 ponctions réalisées en France durant l'année 1999, 18000 l'ont été dans le cadre d'une ICSI et 23000 dans le cadre d'une FIV classique. Parallèlement, on observe une baisse importante du nombre de cycles d'IAD qui est passé de 17000 en 1994 à 8000 en 1998 (tableau 4). La figure 4 reprend les chiffres des tableaux 2 et 4 et ceux de la figure 3, pour présenter l'évolution du nombre de FIV classiques, d'ICSI et d'IAD sur la période 1986-1999. On y observe le développement rapide de l'ICSI entre 1994 et 1998, avec en parallèle, une légère baisse du nombre de FIV classiques et une baisse importante du nombre d'IAD. D'un point de vue purement économique, cette rapide et importante diffusion de l'ICSI au détriment de l'IAD est paradoxale puisque l'IAD a un rapport coût-efficacité nettement plus avantageux que 1'ICSI (Granberg et al., 1996; Philips et al., 2000). Cette question du rapport coût-efficacité est peu discutée en France où les cri-

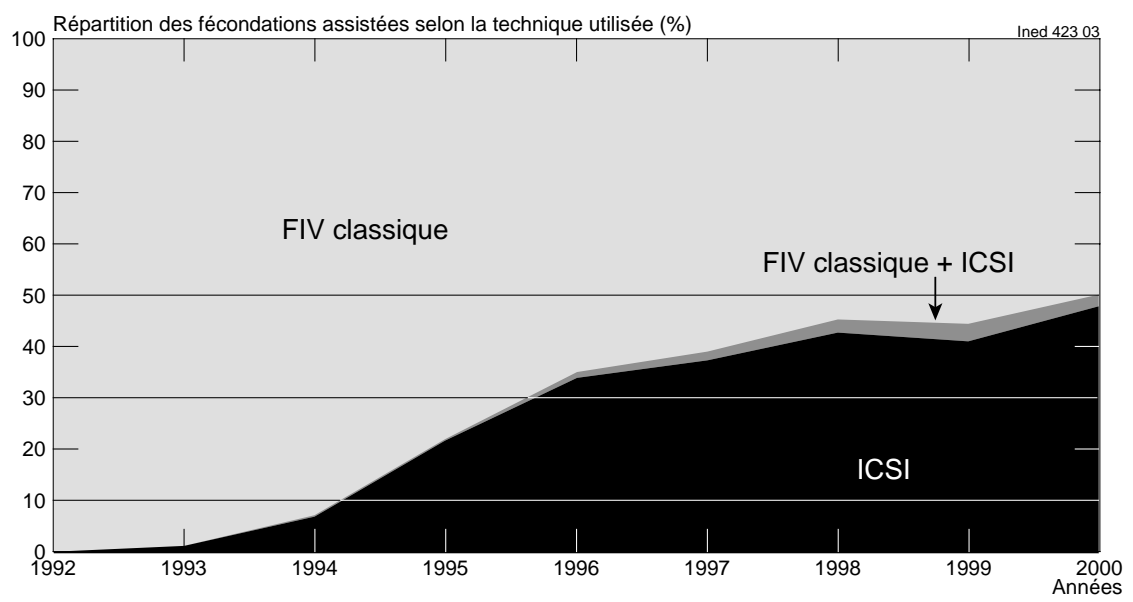

Figure 3.- Part des FIV classiques et des ICSI parmi les fécondations in vitro enregistrées dans FIVNAT entre 1992 et 2000 (en \%)

Note : quelques centres pratiquent des cycles mixtes (ICSI + FIV classique). Dans ce cas, une partie des ovocytes sont fécondés selon la technique de FIV classique et une partie selon la technique d'ICSI. D'après le Guide des bonnes pratiques cliniques et biologiques en Assistance Médicale à la Procréation (CNMBRDP, 1999), ces cycles mixtes doivent rester exceptionnels et documentés dans le dossier médical. De plus, si des embryons sont obtenus par les deux techniques, les embryons obtenus par FIV classique doivent être transférés en priorité Les embryons obtenus par FIV classique et par ICSI ne doivent pas être transférés simultanément (sauf justification dans le dossier médical), en raison notamment de la nécessité d'avoir un suivi correctement documenté des enfants qui en sont issus.

Source : FIVNAT. 


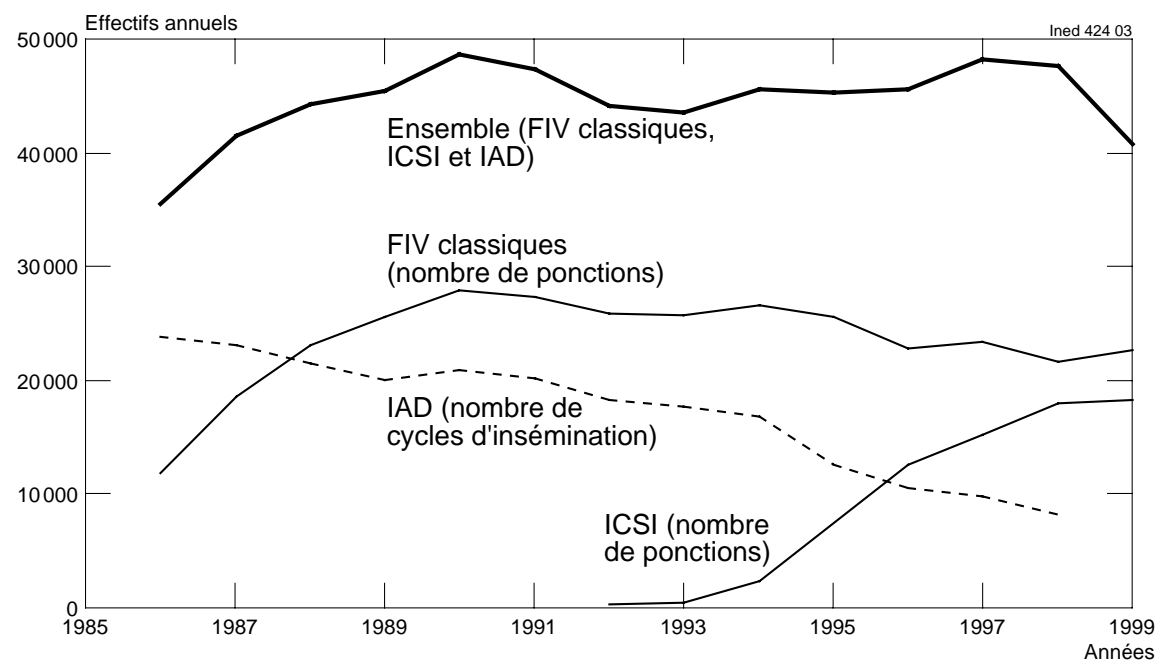

Figure 4.- Nombre de FIV classiques, d'ICSI et d'IAD réalisées en France entre 1986 et 1999

Sources : le nombre annuel d'IAD (nombre de cycles d'insémination) est issu des bilans annuels de la fédération des CECOS. Le nombre annuel de ponctions (englobant FIV classiques et ICSI) est issu des bilans d'activité des centres. L'estimation du nombre de FIV classiques et du nombre d'ICSI est réalisée en appliquant la proportion annuelle d'ICSI observée dans l'enquête FIVNAT.

tères économiques ne pèsent pas sur le choix des couples, qui bénéficient d'une prise en charge des traitements de l'infécondité par le régime de sécurité sociale. Il n'en va pas de même dans tous les pays. Ainsi, aux États-Unis, le coût de l'ICSI en interdit l'accès à une partie de la population qui recourt «par défaut » à l'IAD (Schover et al., 1996). Cela a conduit les médecins américains à souligner qu'avec l'ICSI, une médecine à deux vitesses s'est développée en matière de traitement de l'infertilité masculine (Schover et al., 1996).

La diffusion de l'ICSI peut être suivie au niveau mondial grâce aux bilans de 1995 et 1998. Sur la figure 5, nous avons représenté les vingt pays (ou groupes de pays) pour lesquels l'information était disponible dans les deux bilans(26). En 1998, la proportion d'ICSI varie globalement entre $30 \%$ et $60 \%$ des FIV. En six années (1992-1998), il y a donc eu un large développement de cette technique dans l'ensemble des pays, mais une forte hétérogénéité subsiste : en 1995, la proportion d'ICSI varie de $1 \%$ (en Russie) à $51 \%$ (en Belgique). Dans la majorité des pays, le recours à cette technique a toutefois progressé selon une même tendance

(26) Seuls quatre de ces vingt pays ou groupes de pays ont déclaré leur activité de manière exhaustive pour ces deux années : la Finlande, la Suisse, l'Amérique latine, l'Australie et la Nouvelle-Zélande. Pour les autres, on suppose que la proportion d'ICSI déclarée dans les centres rapportant leur activité est identique à celle des centres ne rapportant par leur activité. 
linéaire(27) : ceux dans lesquels la proportion d'ICSI était de l'ordre de $20 \%$ en 1995 ont atteint une proportion de l'ordre de $42 \%$ en 1998 (Grèce, États-Unis, France, Finlande). Quelques pays s'écartent de cette tendance : en Russie et aux Pays-Bas, la proportion d'ICSI a plus faiblement augmenté, tandis qu'en Hongrie, en Italie et en Espagne, elle s'est accrue davantage. L'Espagne est le pays le plus atypique dans ce domaine, mais la forte progression enregistrée porte sur quatre années au lieu de trois pour les autres pays.

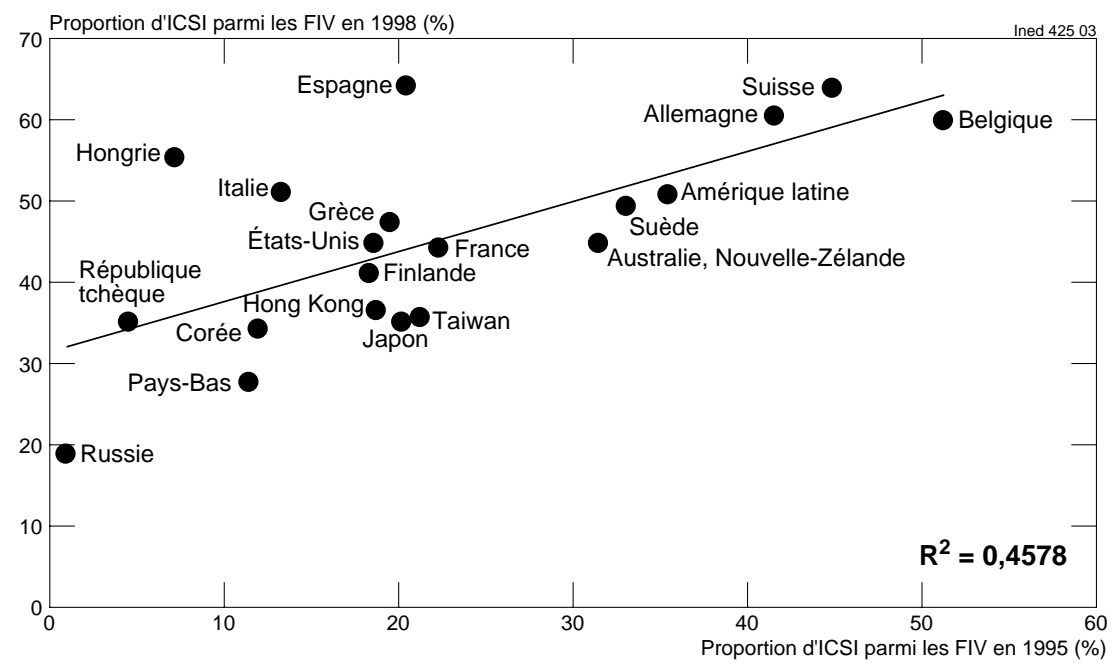

Figure 5.- Proportion d'ICSI parmi les FIV réalisées en 1995 et en 1998 dans vingt pays (en \%)

Source : bilans mondiaux (cf. note 10).

\section{Et demain? Le débat médical autour de la diffusion de l'ICSI}

Ces chiffres mettent en évidence une diffusion rapide et importante de l'ICSI qui conduit à s'interroger sur les raisons et les conditions de l'essor de cette nouvelle technique. D'un point de vue médical, le recours à l'ICSI se justifie dans le cadre d'indications strictes basées sur une altération sévère des caractéristiques spermatiques (Khorram et al., 2001). Cette technique est également proposée en cas d'échec des FIV classiques. Cependant, la question des indications de l'ICSI fait l'objet d'un débat

(27) En excluant les cinq pays les plus atypiques (Russie, Pays-Bas, Hongrie, Italie, Espagne), on estime que dans les quinze autres pays, le recours à l'ICSI a évolué selon une droite d'équation : proportion d'ICSI en $1998(\%)=0,69 \times$ proportion d'ICSI en $1995(\%)+28$. 
scientifique auquel participe activement la revue européenne de reproduction, Human Reproduction (Fishel et al., 2000; Hamilton et Bhattacharya, 2001 ; Ola et al., 2001; Oehninger et Gosden, 2002). Ce débat oppose les partisans du «tout ICSI » qui préconisent un large recours à l'ICSI, même lorsque l'homme présente un sperme normal, et les partisans d'un recours à l'ICSI pour des indications plus restrictives (infertilité masculine, échec de la technique de FIV classique) ${ }^{(28)}$. Ces discussions pourraient expliquer en partie les variations du recours à l'ICSI d'un pays à l'autre. En France, d'après les données FIVNAT, celle-ci répond très majoritairement (dans $71 \%$ des cas pour l'année 2000) à des indications masculines, associées ou non à une indication féminine. Pour le reste, les auteurs du bilan FIVNAT indiquent qu'il s'agit probablement d'ICSI pratiquées après échec de tentatives de FIV.

Les partisans du «tout ICSI »s'appuient sur des travaux indiquant des taux de succès des ICSI supérieurs à ceux des FIV, tandis que les partisans d'un recours plus restrictif à l'ICSI discutent la validité de ces résultats en mettant en avant des problèmes méthodologiques (en particulier des problèmes de biais de sélection). Ces derniers notent par ailleurs l'importance du coût financier de l'ICSI par rapport à la FIV classique (de l'ordre de $+30 \%$ ) et soulignent la nécessité de rester vigilant face à une technique nouvelle dont il faut encore évaluer les conséquences (Cummins et Jequier, 1995 ; Patrizio, 1995 ; Oehninger, 2001 ; Oehninger et Gosden, 2002).

\section{Questions autour de la santé des enfants nés par ICSI}

Dans l'évaluation des conséquences de l'ICSI, la question de l'état de santé des enfants est au cœur des préoccupations. Cette question avait déjà été soulevée lors de la diffusion de la FIV et l'on retrouve par exemple, dans le cas de l'ICSI, les mêmes problèmes liés aux grossesses multiples (avec les risques de mortalité fœto-infantile qui en découlent). Cependant avec l'ICSI, trois interrogations supplémentaires doivent être prises en compte : le fait d'utiliser des spermatozoïdes sévèrement anormaux risque-t-il d'avoir des conséquences pour la descendance? La technique de l'ICSI, plus invasive que celle de la FIV classique (puisque l'ovocyte est pénétré par une micro-pipette(29), augmente-t-elle ces

(28) En France, le Guide des bonnes pratiques cliniques et biologiques en Assistance Médicale à la Procréation (CNMBRDP, 1999) note que l'ICSI doit être réservée à des cas où la FIV classique est impossible ou a de faibles chances de réussite, c'est-à-dire en particulier en cas d'infertilité masculine sévère. Par ailleurs, le Guide indique que l'ICSI peut être envisagée en cas d'échec de la FIV classique. Dans tous les cas, le choix de la technique et ses raisons doivent être clairement explicités dans le dossier médical.

(29) On constate des dommages ovocytaires dans environ $10 \%$ des cas après injection du spermatozoïde. Cf. le Guide des bonnes pratiques cliniques et biologiques en Assistance Médicale à la Procréation (CNMBRDP, 1999). 
risques? Le fait de contourner le processus naturel de sélection des spermatozoïdes, en choisissant un unique spermatozoïde pour la fécondation, peut-il avoir des conséquences néfastes? Afin d'évaluer l'état de santé des enfants, plusieurs indicateurs doivent être utilisés : le risque de malformation congénitale, le développement psychomoteur et l'état de santé à long terme. Différentes «cohortes »d'enfants nés par ICSI ont été constituées en Belgique (Bonduelle et al., 1998 et 2002), en Angleterre (Sutcliffe et al., 2001), en Suède (Wennerholm et al., 2000), au Danemark (Loft et al., 1999), en France (Epelboin et al., 1998), en Australie (Bowen et al., 1998; Hansen et al., 2002) et aux États-Unis (Palermo et al., 1996). Certaines études obtiennent des résultats rassurants (Palermo et al., 1996; Bonduelle et al., 1998 et 2002), mais d'autres concluent à un retard du développement des enfants conçus par ICSI (Bowen et al., 1998) et à un risque accru de malformation congénitale (Kurinczuk et Bower, 1997). Enfin, des auteurs avancent l'hypothèse d'un risque accru de malformation congénitale pour certaines pathologies spécifiques, en particulier celles de l'appareil génital chez les garçons (Wennerholm et al., 2000; Ericson et Kallen, 2001; Sutcliffe et al., 2001) et des anomalies du nombre de chromosomes sexuels (In't Veld et al., 1995 ; Liebaers et al., 1995). Ces résultats discordants reflètent les difficultés méthodologiques rencontrées : difficultés liées aux faibles effectifs observés (en particulier pour mettre en évidence des risques accrus de malformations congénitales rares ${ }^{(30)}$ ), aux taux élevés d'enfants perdus de vue, à la constitution d'un groupe témoin adéquat, au coût financier du suivi d'une cohorte d'enfants nés par fécondation assistée, à des considérations éthiques entourant le suivi d'une cohorte d'enfants et à une hétérogénéité des systèmes de classification des malformations (Mitchell, 1997; Hawkins et Barratt, 1999; Sutcliffe et al., 2001; Sutcliffe, 2002). Devant ces questions, le Guide des bonnes pratiques cliniques et biologiques en Assistance Médicale à la Procréation (CNMBRDP, 1999) souligne que les praticiens se doivent d'informer les couples sur l'état encore limité des connaissances relatives à la santé des enfants et sur les possibles risques liés à la pratique de l'ICSI.

Début mars 2002, une étude relançait plus largement le débat en concluant que les enfants nés par FIV classique et par ICSI ont un risque de malformation congénitale deux fois plus élevé que les enfants nés sans assistance médicale à la procréation (Hansen et al., 2002). Quelques mois plus tard, le British Medical Journal publiait un éditorial proposant la mise en place rapide d'une vaste étude prospective visant à suivre l'état de santé des enfants nés par assistance médicale à la procréation (Sutcliffe, 2002). En France, le comité d'éthique $(\mathrm{CCN})^{(31)}$ remarquait dans son 75e avis publié en décembre 2002 (Comité consultatif national d'éthique

(30) Si l'on considérait une malformation congénitale touchant 1 enfant sur 1000 , il faudrait observer 20000 enfants nés par ICSI et 20000 enfants témoins pour mettre en évidence un doublement de ce risque (Hawkins et al., 1999).

(31) Le Comité consultatif national d'éthique pour les sciences de la vie et de la santé $(\mathrm{CCN})$ dispose d'un site internet où ses avis sont publiés : http://www.ccne-ethique.fr 
pour les sciences de la vie et de la santé (CNNE), 2002) la nécessité de mieux évaluer les conséquences de l'ICSI pour la santé des enfants tout en soulignant que la mise en place d'une cohorte posait des problèmes éthiques, s'inquiétant à la fois d'un risque de stigmatisation des enfants et du respect des libertés individuelles (qui impose un suivi basé sur le volontariat). Les premières recherches sur l'état de santé des enfants nés par ICSI porteront probablement sur les risques de malformation congénitale et sur le développement psychomoteur des enfants. Mais, à plus long terme, d'autres questions devront être soulevées : dans une vingtaine d'années, les premiers enfants nés par ICSI auront 30 ans. Il faudra alors suivre leur fécondité et l'on devra plus spécifiquement s'interroger sur la fertilité des garçons dont le père présentait une infertilité sévère(32). Les fils nés par ICSI auront-ils un risque accru d'être à leur tour des hommes médicalement assistés pour procréer?

\section{Questions autour de la santé des femmes}

La question des conséquences de l'ICSI pour la santé des femmes est également posée. En réalité, cette question concerne plus largement la technique de fécondation in vitro, qu'il s'agisse de FIV classiques ou d'ICSI (Laborie, 1994b). Cependant, dans le cadre de l'ICSI, l'interrogation porte sur l'éthique et la cohérence d'une démarche médicale où les risques de la technique sont supportés par la femme alors que celle-ci présente une fertilité normale, l'infécondité étant liée à son partenaire (Athéa, 1990). Si cette question de la prise en charge de l'infertilité masculine dans le corps de la femme ${ }^{(33)}$ se posait dans le cadre de l'IAD, les conséquences possibles pour la santé des femmes sont plus importantes avec l'ICSI et les interrogations également. Il est intéressant de noter que, même conscientes de ces risques, les conjointes d'hommes infertiles marquent la même préférence que les hommes pour l'ICSI quand elles sont confrontées au choix entre ICSI et IAD(34) (Schover et al., 1996).

En matière de santé des femmes, la question de la santé mentale doit aussi être abordée. L'infécondité est source d'une profonde détresse psychologique et les études ont montré que, dans ce domaine, le facteur prépondérant n'était pas l'implication de l'individu dans le problème d'infécondité (origine féminine ou/et masculine de l'infécondité) mais le sexe de l'individu (Greil, 1997). Même dans le cas d'une infertilité mascu-

(32) Cette question est plus spécifiquement soulevée dans le cas d'une infertilité masculine car on pense que celle-ci pourrait être principalement d'origine génétique, transmise par le père mais également par la mère (Meschede et al., 2000; Ford, 2001). La naissance d'enfants issus d'hommes infertiles grâce à l'assistance médicale à la procréation amène donc à s'interroger sur la transmission de cette infertilité à la génération suivante.

(33) Cette relation entre le corps (en particulier féminin) et les techniques d'assistance médicale à la procréation fait l'objet de réflexions sociologiques (Rouch, 1995; Oudshoorn, 2000; Tain, 2003a).

(34) Cependant, les femmes mentionnent plus souvent que leur conjoint des craintes pour leur santé (Schover et al., 1996). 
line, les femmes sont dans une situation de détresse psychologique plus importante que leur partenaire. Le poids des techniques (IAD, FIV et ICSI), majoritairement supporté par les femmes, pourrait être un élément déterminant pour expliquer cette plus grande détresse féminine (Wright et al., 1991). D'autres explications, plus sociologiques, sont également mises en avant avec l'hypothèse que la reproduction reste le domaine des femmes (Héritier-Augé, 1985 ; Wright et al., 1991), même si l'on sait désormais que l'infécondité n'est pas uniquement d'origine féminine. Enfin, dans ce domaine de la santé mentale, la gestion d'un stress chronique tel que l'infécondité serait différente selon le sexe : les femmes y répondraient en « ruminant» la situation (développant ainsi des réactions de stress et de détresse) alors que la réponse masculine se manifesterait par l'oubli et la négation du problème, en s'investissant dans d'autres domaines (Wright et al., 1991).

\section{Conclusion}

Le monde médical a longtemps considéré que la prise en charge de l'infertilité masculine restait limitée, en particulier face aux succès obtenus pour répondre aux infertilités féminines (Hamberger et Janson, 1997). En cas d'infertilité masculine sévère, le recours à un donneur de sperme a d'abord constitué la seule technique efficace d'assistance médicale à la procréation. Avec l'apparition de l'ICSI, on a donc assisté depuis une décennie à une véritable révolution dans la prise en charge médicale de l'infertilité masculine : l'homme infertile peut désormais concevoir in vitro un enfant qui est génétiquement le sien. Si la rapide et importante diffusion de l'ICSI traduit le succès d'une technique d'assistance médicale à la procréation développée pour répondre aux infertilités masculines, elle ne va pas sans soulever des questions. Ses limites sont économiques (coût important de l'ICSI), éthiques (prise en charge médicale de l'infertilité masculine dans le corps de la femme) et sanitaires, avec en particulier des questions autour de la santé, à court et à long terme, des enfants nés par ICSI. Face à ces discussions, gardons à l'esprit que d'autres révolutions médicales pourraient venir bouleverser la prise en charge médicale de l'infertilité masculine à l'avenir, tant sur le plan du diagnostic que sur celui de son traitement (Ford, 2001).

Remerciements. Ma gratitude va à Jacques de Mouzon (Inserm) qui, depuis plus d'une année, suit mon travail sur les données d'assistance médicale à la procréation; la réalisation de cet article doit beaucoup à cette collaboration. Je remercie également l'ensemble de l'équipe FIVNAT qui gère, collecte et saisit les données de l'enquête, sans laquelle cette analyse de la fécondation assistée en France n'aurait pu être effectuée.

Je remercie Henri Leridon (Ined-Inserm), Alfred Spira (AP-HP) et Annie Bachelot (Inserm) pour leurs remarques constructives lors de la relecture des premières épreuves de ce travail. Ma gratitude va enfin à Loïc Desquilbet pour son aide technique à la conception des graphiques sur les données européennes et mondiales. 


\section{RÉFÉRENCES}

AthÉA N., 1994, «La stérilité : une entité mal définie », in J. Testart (dir.), Le magasin des enfants, Gallimard (Coll. Folio), p. 47-95.

Baird D.D., Weinberg C.R., Schwingl P., Wilcox A.J., 1994, « Selection bias associated with contraceptive practice in time-to-pregnancy studies », Annals of the New York Academy of Sciences, 709, p. 156-164.

Bonduelle M., Joris H., Hofmans K., Liebaers I., Van Steirteghem A., 1998, « Mental development of 201 ICSI children at 2 years of age », The Lancet, 351(9115), p. 1553.

Bonduelle M., Liebaers I., Deketelaere V., Derde M.P., Camus M., Devroey P., VAN STEIRTEGHEM A., 2002, « Neonatal data on a cohort of 2889 infants born after ICSI (1991-1999) and of 2995 infants born after IVF (1983-1999) », Human Reproduction, 17(3), p. 671-694.

Bowen J.R., Gibson F.L., LeSLIE G.I., SAUnders D.M., 1998, « Medical and developmental outcome at 1 year for children conceived by intracytoplasmic sperm injection », The Lancet, 351(9115), p. 1529-1534.

Braverman A.M., CoRSON S.L., 1995, «Factors related to preferences in gamete donor sources », Fertility and Sterility, 63(3), p. 543-549.

Breart G., Mouzon J. de, 1995, « AMP vigilance », Bulletin de l'Académie nationale de médecine, 179(8), p. 1759-1764.

Carlsen E., Giwercman A., Keiding N., Skakkebaek N.E., 1992, « Evidence for decreasing quality of semen during past 50 years », British Medical Journal, 305(6854), p. 609-613.

Charbit Y., 1989, « L'opinion sur la politique démographique, la nuptialité et les nouvelles techniques de procréation en mai 1987 », Population, 44(6), p. 1159-1187.

CNMBRDP (Commission nationale de médecine et de biologie de la reproduction et du diagnostic prénatal), 1999, Guide des bonnes pratiques cliniques et biologiques en Assistance Médicale à la Procréation, Journal Officiel de la République Française du 28 février 1999 (arrêté du 12 janvier 1999).

COMItÉ CONSUlTATIF NATIONAL D'ETHIQUE POUR LES SCIENCES DE LA VIE ET DE LA SANTÉ (CNNE), 2002, Questions éthiques soulevées par le développement de l'ICSI, Avis du CNNE, 75, $11 \mathrm{p}$.

Crosignani P.G., Rubin B., Acosta A., Benagiono G., Collins J., Diczfalusy E., Farley T., Hargreave T., Tarlatzis B., Van Steirteghem A., Walters E., Diedrich K., Ragni G., 1994, « Male sterility and subfertility: guidelines for management. The ESHRE Capri Workshop Group », Human Reproduction, 9(7), p. 1260-1264.

Cummins J.M., Jequier A.M., 1995, «Concerns and recommendations for intracytoplasmic sperm injection (ICSI) treatment », Human Reproduction, 10(Suppl. 1), p. 138-143.

DAGUET F., 2002, Un siècle de fécondité française : caractéristiques et évolution de la fécondité de 1901 à 1999 (Insee Résultats, 8), 305 p.

Dehan M., 1998, « La part des traitements de l'infertilité dans la grande prématurité en France. L'avis du néonatologiste », Contraception, Fertilité, Sexualité, 26(7-8), p. 512-516.

Enginsu M.E., Pieters M.H., Dumoulin J.C., Evers J.L., Geraedts J.P., 1992, « Male factor as determinant of in-vitro fertilization outcome », Human Reproduction, 7(8), p. 11361140 .

Epelboin S., Blondeau M.-A., 1989, «Grossesses multiples après procréation médicalement assistée : devenir obstétrical », Contraception, Fertilité, Sexualité, 17(7-8), p. 756-758.

Epelboin S., Coffineau A., lucas H., De Medeiros N., Francoual C., Goujeard J., Poirot C., Izard V., Thiounn N., Abirached F., Quantin P., Douard S., Ventadour M., JOUANNET P., ZORN J.R., WOLF J.P., 1998, « Exhaustive assessment of 150 post-ICSI pregnancies and subsequent children's development (1994-1996) », Human Reproduction, 13(Abstract book), p. 190-191.

ERICSON A., KALlen B., 2001, «Congenital malformations in infants born after IVF: a population-based study », Human Reproduction, 16(3), p. 504-509.

ESHRE CAMPUS COURSE REPORT, 2001, « Prevention of twin pregnancies after IVF/ICSI by single embryo transfer », Human Reproduction, 16(4), p. 790-800.

FÉdÉrATION FRANÇAISE DES CECOS, LE LANNOU D., LANSAC J., 1989, « Artificial procreation with frozen donor semen: experience of the French Federation CECOS », Human Reproduction, 4(7), p. 757-761. 
Fishel S., Aslam I., Lisi F., Rinaldi L., Timson J., Jacobson M., Gobetz L., Green S., CAmpbell A., Lisi R., 2000, « Should ICSI be the treatment of choice for all cases of invitro conception? », Human Reproduction, 15(6), p. 1278-1283.

FIVNAT, Piette C., Mouzon J. de, Bachelot A., Spira A., 1990, « In-vitro fertilization: influence of women's age on pregnancy rates », Human Reproduction, 5(1), p. 56-59.

FIVNAT, Mouzon J. de, Bachelot A., 1991, «Âge des femmes et fécondation in vitro », Contraception, Fertilité, Sexualité, 19(7-8), p. 567-569.

FIVNAT, 1993, «French National IVF Registry: analysis of 1986 to 1990 data », Fertility and Sterility, 59(3), p. 587-595.

FIVNAT, 1995, « Pregnancies and births resulting from in vitro fertilization: French national registry, analysis of data 1986 to $1990 »$, Fertility and Sterility, 64(4), p. 746-756.

FiVnat, Belaisch-Allart J., Mouzon J. de, Bachelot A., De Vecchi A., Renon C., 1997, «Âge et PMA », Contraception, Fertilité, Sexualité, 25(7-8), p. 503-506.

Ford W.C., 2001, «Biological mechanisms of male infertility », The Lancet, 357(9264), p. $1223-1224$.

Garceau L., Henderson J., Davis L.J., Petrou S., Henderson L.R., McVeigh E., Barlow D.H., DAVIDSON L.L., 2002, «Economic implications of assisted reproductive techniques: a systematic review », Human Reproduction, 17(12), p. 3090-3109.

Giorgetti C., 1987, « Spermogramme et chances de succès de la fécondation in vitro », Contraception, Fertilité, Sexualité, 15(7-8), p. 703.

Granberg M., Wikland M., Hamberger L., 1996, «Cost-effectiveness of intracytoplasmic sperm injection in comparison with donor insemination », Acta Obstetricia et Gynecologica Scandinavica, 75(8), p. 734-737.

GreIL A.L., 1997, «Infertility and psychological distress: a critical review of the literature », Social Science \& Medicine, 45(11), p. 1679-1704.

HAMBERGER L., JANSON P.O., 1997, « Global importance of infertility and its treatment: role of fertility technologies », International Journal of Gynaecology and Obstetrics, 58(1), p. 149-158.

Hamilton M., Bhattacharya S., 2001, « Should ICSI be the treatment for all cases of in-vitro conception? », Human Reproduction, 16(4), p. 801-802.

Hansen M., Kurinczuk J.J., Bower C., Webb S., 2002, « The risk of major birth defects after intracytoplasmic sperm injection and in vitro fertilization », New England Journal of Medicine, 346(10), p. 725-730.

HAWkins M.M., BARRATT C.L., 1999, « Intracytoplasmic sperm injection », Science, 286(5437), p. $51-52$.

Hawkins M.M., Barratt C.L., Sutcliffe A.G., Cooke I.D., 1999, « Male infertility and increased risk of diseases in future generations », The Lancet, 354(9193), p. 1906-1907.

HÉritier-Augé F., 1985, «La cuisse de Jupiter: réflexions sur les nouveaux modes de procréation », L'Homme, 25(94), p. 5-22 (publié à nouveau dans F. Héritier, 1996, Masculin - Féminin. La pensée de la différence, Paris, Odile Jacob, p. 253-275).

Hull M.G., Eddowes H.A., Fahy U., Abuzeid M.I., Mills M.S., Cahill D.J., Fleming C.F., WARdle P.G., Ford W.C., MCDERMOTT A., 1992, « Expectations of assisted conception for infertility », British Medical Journal, 304(6840), p. 1465-1469.

Humeau C., Arnal F., 1994, Les médecines de procréation : comptes et mécomptes de la fécondation in vitro, Éditions Odile Jacob, 407 p.

In'T Veld P., Brandenburg H., VerhoefF A., Dhont M., Los F., 1995, « Sex chromosomal abnormalities and intracytoplasmic sperm injection », The Lancet, 346(8977), p. 773.

IRvine D.S., 1998, «Epidemiology and aetiology of male infertility », Human Reproduction, 13(Suppl. 1), p. 33-44.

Khorram O., Patrizio P., WANG C., Swerdloff R., 2001, « Reproductive technologies for male infertility », Journal of Clinical Endocrinology and Metabolism, 86(6), p. 2373-2379.

KURINCZUK J.J., BowER C., 1997, « Birth defects in infants conceived by intracytoplasmic sperm injection: an alternative interpretation », British Medical Journal, 315(7118), p. 12601265 ; discussion p. 1265-1266.

LABORIE F., 1994a, « Nouvelles technologies de la reproduction (NTR) : risques pour la santé des enfants », in Les modes de régulation de la reproduction humaine : incidences sur la fécondité et la santé, actes du colloque international de l'Aidelf, Delphes, 1992, p. 771777.

LABORIE F., 1994b, «Nouvelles technologies de la reproduction (NTR) : risques pour la santé des femmes », in Les modes de régulation de la reproduction humaine : incidences sur 
la fécondité et la santé, actes du colloque international de 1'Aidelf, Delphes, 1992, p. 757-770.

LAMBERT R.D., 2002, «Safety issues in assisted reproduction technology: The children of assisted reproduction confront the responsible conduct of assisted reproductive technologies », Human Reproduction, 17(12), p. 3011-3015.

LERIDON H., 1980, "La stérilité et l'insémination artificielle par donneur dans l'opinion », Contraception, Fertilité, Sexualité, 8(2), p. 153-158.

LERIDON H., 1981, «Fécondité, fertilité, stérilité : problèmes de terminologie », in A. Spira, P. Jouannet (dir.), Facteurs de la fertilité humaine (avec référence particulière au sexe masculin), les Colloques de l'Inserm, Cargèse, 21-23 septembre 1981, p. 17-30.

Leridon H., SpIRA A., 1984, « Problems in measuring the effectiveness of infertility therapy », Fertility and Sterility, 41(4), p. 580-586.

Leridon H., Charbit Y., Collomb P., SARdon J.-P., Toulemon L. (éd.), 1987, La seconde révolution contraceptive. La régulation des naissances en France de 1950 à 1985 (Travaux et Documents, Cahier n ${ }^{\circ} 117$ ), Ined, 380 p.

LERIDON H., 1990, « De la procréation naturelle à la procréation médicalement assistée : le point de vue du démographe », Journal de la Société de Statistique de Paris, 131(2), p. 3-23.

LERIDON H., 1991, « Stérilité et hypofertilité : du silence à l'impatience? », Population, 46(2), p. 227-248.

Leridon H., Oustry P., Bajos N., ÉQuipe Cocon, 2002, « La médicalisation croissante de la contraception en France », Population et Sociétés, $\mathrm{n}^{\circ} 381$.

LÉvy C., 1994, «Aperçu d'une population ayant eu recours à la procréation médicalement assistée ", in Les modes de régulation de la reproduction humaine : incidences sur la fécondité et la santé, actes du colloque international de l'Aidelf, Delphes, 1992, p. 353 364 .

Liebaers I., Bonduelle M., Van Assche E., Devroey P., Van Steirteghem A., 1995, « Sex chromosome abnormalities after intracytoplasmic sperm injection », The Lancet, 346(8982), p. 1095.

Loft A., Petersen K., Erb K., Mikkelsen A.L., Grinsted J., Hald F., Hindkjaer J., Nielsen K.M., Lundstrom P., Gabrielsen A., Lenz S., Hornnes P., Ziebe S., Ejdrup H.B., Lindhard A., Zhou Y., Nyboe andersen A., 1999, « A Danish national cohort of 730 infants born after intracytoplasmic sperm injection (ICSI) 1994-1997 », Human Reproduction, 14(8), p. 2143-2148.

Madani-Perret F.Z., 1987, «Le don de sperme : la règle de l'anonymat », Contraception, Fertilité, Sexualité, 15(7-8), p. 678-680.

MAhlstedt P.P., GReENFELd D.A., 1989, «Assisted reproductive technology with donor gametes: the need for patient preparation », Fertility and Sterility, 52(6), p. 908-914.

Mansour R.T., Aboulghar M.A., Serour G.I., Amin Y.M., Ramzi A.M., 1995, « The effect of sperm parameters on the outcome of intracytoplasmic sperm injection », Fertility and Sterility, 64(5), p. 982-986.

Marcus-SteifF J., 1990, "Les taux de succès de la FIV, fausses transparences et vrais mensonges », La Recherche, 21(225), p. 1300-1312.

Marcus-STEIFF J., 1991, «La controverse sur les taux de "succès" de la FIV », La Recherche, 22(231), p. 524-529.

MARCus-STEIFF J., 1994a, «La mesure des taux de "succès" de la FIV », in Les modes de régulation de la reproduction humaine : incidences sur la fécondité et la santé, actes du colloque international de 1'Aidelf, Delphes, 1992, p. 637-647.

MARCus-STEIFF J., 1994b, «Les procréations artificielles : inventaire des techniques, effets sur la natalité », in Les modes de régulation de la reproduction humaine : incidences sur la fécondité et la santé, actes du colloque international de l'Aidelf, Delphes, 1992, p. 365385 .

Mazzone J.-C., 2000, «L'anonymat garant de la levée du secret de la conception dans 1'I.A.D. », Reproduction humaine et hormones, 13(5), p. 528-531.

Meschede D., Lemcke B., Behre H.M., De Geyter C., Nieschlag E., Horst J., 2000, «Clustering of male infertility in the families of couples treated with intracytoplasmic sperm injection », Human Reproduction, 15(7), p. 1604-1608.

Mitchell A.A., 1997, «Intracytoplasmic sperm injection: offering hope for a term pregnancy and a healthy child? », British Medical Journal, 315(7118), p. 1245-1246.

Mouzon J. de, SpIRA A., Schwartz D., 1988, « A prospective study of the relation between smoking and fertility », International Journal of Epidemiology, 17(2), p. 378-384. 
Mouzon J. de, Bachelot A., SPIRA A., 1993, « Establishing a national in vitro fertilization registry: methodological problems and analysis of success rates », Statistics in Medicine, 12(1), p. 39-50.

Mouzon J. de, Thonneau P., Spira A., Multigner L., 1996, « Declining sperm count. Semen quality has declined among men born in France since $1950 »$, British Medical Journal, 313(7048), p. 43; discussion p. 44-45.

Netter A., Belaisch J., 1991, « Histoire de la procréation médicalement assistée », Reproduction humaine et hormones, 3(5), p. 283-293.

Nygren K.G., Andersen A.N., 2001a, « Assisted reproductive technology in Europe, 1997. Results generated from European registers by ESHRE. European IVF-Monitoring Programme (EIM) for the European Society of Human Reproduction and Embryology (ESHRE) », Human Reproduction, 16(2), p. 384-391.

Nygren K.G., ANDERSEN A.N., 2001b, « Assisted reproductive technology in Europe, 1998. Results generated from European registers by ESHRE », Human Reproduction, 16(11), p. 2459-2471.

Oehninger S., Veeck L., Lanzendorf S., Maloney M., Toner J., Muasher S., 1995 , «Intracytoplasmic sperm injection: achievement of high pregnancy rates in couples with severe male factor infertility is dependent primarily upon female and not male factors », Fertility and Sterility, 64(5), p. 977-981.

OEHNINGER S., 2001, «Place of intracytoplasmic sperm injection in management of male infertility », The Lancet, 357(9274), p. 2068-2069.

Oehninger S., Gosden R.G., 2002, « Should ICSI be the treatment of choice for all cases of invitro conception?: No, not in light of the scientific data », Human Reproduction, 17 (9), p. 2237-2242.

Ola B., Afnan M., Sharif K., Papaioannou S., Hammadieh N., Barratt C.L., 2001, « Should ICSI be the treatment of choice for all cases of in-vitro conception? Considerations of fertilization and embryo development, cost effectiveness and safety », Human Reproduction, 16(12), p. 2485-2490.

Olivennes F., 2001, «Grossesse gémellaire après traitement de l'infertilité : échec ou succès? », Gynécologie obstétrique \& fertilité, 29(3), p. 257-260.

Olsen J., Juul S., Basso O., 1998, « Measuring time to pregnancy. Methodological issues to consider », Human Reproduction, 13(7), p. 1751-1753.

Oudshoorn N., 2000, «Au sujet des corps, des techniques et des féminismes », in D. Gardey, I. Löwy (dir.), L'invention du naturel : les sciences et la fabrication du féminin et du masculin. Histoire des sciences, des techniques et de la médecine, Paris, Éditions des archives contemporaines, p. 31-44.

Palermo G., Joris H., Devroey P., Van Steirteghem A.C., 1992, « Pregnancies after intracytoplasmic injection of single spermatozoon into an oocyte », The Lancet, 340(8810), p. 1718.

Palermo G.D., Colombero L.T., Schattman G.L., Davis O.K., Rosenwaks Z., 1996, « Evolution of pregnancies and initial follow-up of newborns delivered after intracytoplasmic sperm injection », Journal of American Medical Association, 276(23), p. 18931897.

PAPIERnik E., Mouzon J. de, 2002, « Triplet pregnancies from French IVF centers: 1986-98 », in L.G. Keith, I. Blickstein (dir.), Triplet pregnancies and their consequences, Parthenon Publishing Group, p. 45-54.

PAtrizio P., 1995, «Intracytoplasmic sperm injection (ICSI): potential genetic concerns », $\mathrm{Hu}$ man Reproduction, 10(10), p. 2520-2523.

Payne D., Flaherty S.P., Jeffrey R., Warnes G.M., Matthews C.D., 1994, « Successful treatment of severe male factor infertility in 100 consecutive cycles using intracytoplasmic sperm injection », Human Reproduction, 9(11), p. 2051-2057.

Philips Z., Barraza-Llorens M., Posnett J., 2000, « Evaluation of the relative cost-effectiveness of treatments for infertility in the UK », Human Reproduction, 15(1), p. 95-106.

Plachot M., 1987, «Les indications masculines de la FIV. Enquête nationale », Contraception, Fertilité, Sexualité, 15(7-8), p. 699-700.

Rojo Moreno J., Valdemoro Garcia C., Garcia Merita M.L., Tortajada Martinez M.G., 1994, «Personality characteristics analysis in couples undergoing artificial insemination », Human Reproduction, 9(1), p. 172-175. 
Rojo Moreno J., Valdemoro C., Garcia Merita M.L., Tortajada M., 1996, « Analysis of the attitudes and emotional processes in couples undergoing artificial insemination by donor », Human Reproduction, 11(2), p. 294-299.

Rouch H., 1995, «Les nouvelles techniques de reproduction: vers l'indifférenciation sexuelle? », in A. Ducros, M. Panoff (dir.), La frontière des sexes (Le Sociologue), Paris, Puf, p. 251-269.

SARdon J.-P., 2002, «Évolution démographique récente des pays développés. », Population, 57(1), p. 123-170.

Schover L.R., Thomas A.J., Miller K.F., Falcone T., Attaran M., Goldberg J., 1996, «Preferences for intracytoplasmic sperm injection versus donor insemination in severe male factor infertility: a preliminary report », Human Reproduction, 11(11), p. 24612464.

Schwartz D., Mayaux M.J., 1982, «Female fecundity as a function of age: results of artificial insemination in 2193 nulliparous women with azoospermic husbands. Federation CECOS », New England Journal of Medicine, 306(7), p. 404-406.

Sharma V., Allgar V., Rajkhowa M., 2002, "Factors influencing the cumulative conception rate and discontinuation of in vitro fertilization treatment for infertility », Fertility and Sterility, 78(1), p. 40-46.

SPIRA A., 1986, « Epidemiology of human reproduction », Human Reproduction, 1(2), p. 111 115 .

Steptoe P.C., Edwards R.G., 1978, « Birth after the reimplantation of a human embryo », The Lancet, 2(8085), p. 366.

Sutcliffe A.G., Taylor B., Saunders K., Thornton S., Lieberman B.A., Grudzinskas J.G., 2001, «Outcome in the second year of life after in-vitro fertilisation by intracytoplasmic sperm injection: a UK case-control study », The Lancet, 357(9274), p. 2080-2084.

Sutcliffe A.G., 2002, "Health risks in babies born after assisted reproduction », British Medical Journal, 325(7356), p. 117-118.

TAIN L., 2001, «L'hôpital, la femme et le médecin : la construction des trajectoires de fécondation in vitro », Population, 56(5), p. 811-844.

TAIN L., 2003a, «Corps reproducteur et techniques procréatives : images, brouillages, montages et remue-ménage », Cahiers du Genre, 34 (numéro spécial "La distinction entre science et genre : une histoire entre biologie et culture"), I. Löwy, H. Rouch (coord.), p. 171-192.

TAIN L., 2003b, «Évaluation et expansion des techniques de reproduction : des succès pour qui ? », Santé publique et Sciences sociales, 10, à paraître.

Tesarik J., Mendoza C., Testart J., 1995, « Viable embryos from injection of round spermatids into oocytes », New England Journal of Medicine, 333(8), p. 525.

Testart J., Frydman R., 1982, « Naissance à terme après implantation intra-utérine d'un embryon obtenu par fécondation in vitro », Journal de Gynécologie, Obstétrique et Biologie de la Reproduction, 11(7), p. 855-859.

Thibault C., Cohen J., Zorn J.R., Cedard L., Hedon B., Salat-Baroux J., Pouly J.-L., Buvat J., Brunetti P.-M., Blanc F., Relier J.-P., Jouhet P., Marcus-Steiff J., 1991, «La controverse sur les taux de succès de la FIV », La Recherche, 22(231), p. 524-529.

Thonneau P., Marchand S., Tallec A., Ferial M.L., Ducot B., Lansac J., Lopes P., TABAste J.-M., SPIRA A., 1991, «Incidence and main causes of infertility in a resident population $(1,850,000)$ of three French regions (1988-1989) », Human Reproduction, 6(6), p. 811-816.

Tournaye H., Devroey P., Camus M., Staessen C., Bollen N., Smitz J., Van Steirteghem A.C., 1992, "Comparison of in-vitro fertilization in male and tubal infertility: a 3 year survey », Human Reproduction, 7(2), p. 218-222.

VAN Den Eede B., 1995, «Investigation and treatment of infertile couples: ESHRE guidelines for good clinical and laboratory practice. European Society of Human Reproduction and Embryology », Human Reproduction, 10(5), p. 1246-1271.

Vayena E., Rowe P.J., Peterson H.B., 2002, « Assisted reproductive technology in developing countries: why should we care ? », Fertility and Sterility, 78(1), p. 13-15.

Weinberg C.R., Dunson D.B., 2000, «Some issues in assessing human fertility », Journal of the American Statistical Association, 95, p. 300-303.

Wennerholm U.B., Bergh C., Hamberger L., Lundin K., Nilsson L., Wikland M., Kallen B., 2000, «Incidence of congenital malformations in children born after ICSI », Human Reproduction, 15(4), p. 944-948. 
Wright J., Duchesne C., Sabourin S., Bissonnette F., Benoit J., Girard Y., 1991, «Psychosocial distress and infertility: men and women respond differently », Fertility and Sterility, 55(1), p. 100-108.

Yovich J.L., Matson P.L., 1995, « Male subfertility: concepts in 1995 », Human Reproduction, 10(Suppl. 1), p. 3-9. 
LA Rochebrochard Élise de.- Des hommes médicalement assistés pour procréer : IAD, FIV, ICSI, bilan d'une révolution dans la prise en charge médicale de l'infertilité masculine

Face aux problèmes d'infertilité, qui concernent $15 \%$ à $20 \%$ des couples, on a assisté au développement des techniques de fécondation in vitro (FIV). Entre 1982 et 2000, 85000 enfants sont nés en France grâce à une FIV (estimation basée sur une exploitation croisée de l'enquête FIVNAT et des données administratives). Cinq bilans mondiaux et deux bilans européens ont été consacrés à la FIV mais leur exploitation reste limitée par les problèmes de non-exhaustivité des données pour des régions telles que l'Europe méridionale et orientale. Les taux de succès de la FIV sont de l'ordre de $15 \%$ à $20 \%$ de grossesses par ponction. Cependant, ces taux chutent quand l'âge de la femme augmente et lorsque l'homme a un sperme présentant des anomalies sévères. Dans le cas d'une infertilité masculine sévère, l'insémination artificielle avec spermatozoïdes de donneur (IAD) a longtemps été la seule réponse médicale, mais elle pose le problème de l'acceptation d'un donneur de sperme. Depuis 1992, une nouvelle technique de FIV est proposée : l'injection intra-cytoplasmique de spermatozoïde (ICSI). Cette technique s'est largement développée en France et dans de nombreux pays : elle représentait $30 \%$ à $60 \%$ de l'activité de fécondation assistée en 1998. Malgré ce développement important, de nombreuses questions demeurent sur les conséquences de la technique, en particulier quant à l'état de santé à court et à long terme des enfants conçus par ICSI.

\section{La Rochebrochard Élise de.- Men Medically Assisted to Reproduce: AID, IVF, and ICSI,} an Assessment of the Revolution in the Medical Treatment of Male Factor Infertility

In response to involuntary infertility, which affects $15 \%$ to $20 \%$ of couples, the techniques of in vitro fertilization (IVF) have been developed. Between 1982 and 2000, 85,000 children were born in France thanks to IVF (estimates based on combined analysis of the FIVNAT survey and administrative data). Five world reports and two European reports have been produced on IVF, but their use remains limited by incomplete data for regions such as southern and eastern Europe. The success rates with IVF are around $15 \%$ to $20 \%$ of pregnancies obtained per retrieval. However, these rates decline rapidly as the woman's age rises and when the man's sperm has severe abnormalities. In cases of severe male factor infertility, artificial insemination by donor (AID) was for long the only medical solution, but it raises the problem of accepting sperm from a donor. Since 1992, a new IVF technique, intracytoplasmic sperm injection (ICSI) is available. This technique has been widely adopted in France and in many other countries: in 1998 it represented $30 \%$ to $60 \%$ of assisted fertilizations. Despite this large development, numerous questions remain concerning the consequences of the technique, particularly regarding the short- and long-term health of children conceived by ICSI.

LA RocheBRochARD Élise de.- Sobre los hombres que recurren a la asistencia médica para procrear: IAD, FIV, ICSI, balance de una revolución en la respuesta médica a la infertilidad masculina

Ante los problemas de infertilidad, que afectan entre el $15 \%$ y el $20 \%$ de las parejas, se ha producido una evolución de las técnicas de fecundación in vitro (FIV). Entre 1982 y el 2000 se produjeron 85,000 nacimientos en Francia gracias a la FIV (estimación basada en una explotación combinada de la encuesta FIVNAT y de datos administrativos). Aunque se han realizado cinco balances del FIV a nivel mundial y dos a nivel europeo, la explotación de tales balances es limitada debido a problemas de falta de exhaustividad de los datos para ciertas regiones, por ejemplo Europa meridional y del Este. Las tasas de éxito de la FIV son del orden del 15 al $20 \%$ de embarazos por punción. No obstante, estas tasas disminuyen fuertemente cuando la edad de la mujer aumenta o cuando el esperma del hombre presenta anomalías severas. En casos de infertilidad masculina severa, la única respuesta médica ha sido durante mucho tiempo la inseminación artificial con espermatozoides del donante (IAD), pero tal método requiere la aceptación del donante de esperma. Desde 1992 existe una nueva técnica de FIV: la inyección intra-citoplásmica de espermatozoide (ICSI). Esta técnica se ha desarrollado ampliamente tanto en Francia como en otros países, hasta representar entre el $30 \%$ y el $60 \%$ de la actividad de fecundación asistida en 1998. A pesar de una evolución tan significativa, todavía existen muchas dudas en cuanto a las consecuencias de ésta técnica, especialmente en cuanto al estado de salud a corto y a largo plazo de los niños concebidos a partir de ella.

Élise de LA Rochebrochard, Unité mixte Ined-Inserm «Épidémiologie, démographie et sciences sociales : santé reproductive, sexualité et infection à VIH », Hôpital de Bicêtre, 82 rue du Général Leclerc, 94276 Le Kremlin-Bicêtre, France, tél. : 01452123 33, fax : 01452120 75, courriel : roche@ined.fr 